Published in The Astronomical Journal (AJ, 143, 103). Available At: http://stacks. iop.org/1538-3881/143/103

Preprint typeset using LATEX style emulateapj v. 12/16/11

\title{
A MULTI-SURVEY APPROACH TO WHITE DWARF DISCOVERY
}

\author{
Conor Sayres ${ }^{1,4,5}$, John P. Subasavage ${ }^{2,4}$, P. Bergeron ${ }^{3}$, P. Dufour ${ }^{3}$, James R. A. Davenport ${ }^{1}$, Yusra \\ AlSAYYAD ${ }^{1}$, And Benjamin M. TOFFlemire ${ }^{1}$ \\ Published in The Astronomical Journal (AJ, 143, 103). Available At: http://stacks. iop. org/1538-3881/143/103
}

\section{ABSTRACT}

By selecting astrometric and photometric data from the Sloan Digital Sky Survey (SDSS), the Lépine \& Shara Proper Motion North Catalog (LSPM-North), the Two Micron All Sky Survey (2MASS), and the USNO-B1.0 catalog, we use a succession of methods to isolate white dwarf candidates for follow-up spectroscopy. Our methods include: reduced proper motion diagram cuts, color cuts, and atmospheric model adherence. We present spectroscopy of 26 white dwarfs obtained from the CTIO $4 \mathrm{~m}$ and APO $3.5 \mathrm{~m}$ telescopes. Additionally, we confirm 28 white dwarfs with spectra available in the SDSS DR7 database but unpublished elsewhere, presenting a total of 54 WDs. We label one of these as a recovered WD while the remaining 53 are new discoveries. We determine physical parameters and estimate distances based on atmospheric model analyses. Three new white dwarfs are modeled to lie within 25 pc. Two additional white dwarfs are confirmed to be metal-polluted (DAZ). Follow-up time series photometry confirms another object to be a pulsating ZZ Ceti white dwarf.

Subject headings: Catalogs - Proper motions - Stars: variables: general - Surveys - white dwarfs

\section{INTRODUCTION}

White dwarfs (WDs) are of interest in a variety of subfields in astrophysics because of their unique ability to act as cosmic chronometers. Theoretical cooling models provide a means for dating WDs from photometry alone, and this process has provided constraints on the age of the Galactic disk using cool WD samples (e.g., Leggett et al. 1998). Because the coolest (and hence oldest) WDs are less luminous, the nearby representatives provide the best opportunities for accurate characterization.

The present understanding of the local WD sample is somewhat uncertain. Holberg et al. (2008) estimate that the WD sample is $\sim 80 \%$ complete to 20 pc. Including only WDs with accurate trigonometric parallaxes, Subasavage et al. (2009) conclude that the sample is only $\sim 47 \%$ complete to $25 \mathrm{pc}$. The vast majority of the incompleteness arises from the coolest WDs that remain to be discovered. Additional discoveries of nearby WDs will strengthen the completeness statistics and provide valuable model parameter constraints through trigonometric parallax measurements.

The Sloan Digital Sky Survey (SDSS; York et al. 2000) has been exceptional at identifying WDs. The lastest release as of the writing of this manuscript, Data Release 8 (DR8; Aihara et al. 2011), reports sky coverage of over $14,500 \mathrm{deg}^{2}$. Imaging data are collected in the ugriz bands with a $50 \%$ completeness limit at $r=22.5$ for point sources, and spectra have been observed for over half of a

\footnotetext{
csayres@u.washington.edu

${ }^{1}$ Department of Astronomy, University of Washington, Box 351580, Seattle, WA 98195

${ }^{2}$ US Naval Observatory, 10391 West Naval Observatory Road, Flagstaff, AZ 86001-8521

3 Département de Physique, Université de Montréal, C.P.6128, Succursale Centre-Ville, Montréal, QC H3C 3J7, Canada

${ }^{4}$ Visiting Astronomer, Cerro Tololo Inter-American Observatory. CTIO is operated by AURA, Inc. under contract to the National Science Foundation.

${ }^{5}$ CTIO REU 2010.
}

million stars. Prior data releases from SDSS have led to a proliferation of WD discoveries. Eisenstein et al. (2006) (hereafter SDSS-E06) roughly doubled the number of previous, spectroscopically identified WDs using spectra from the DR4 database Adelman-McCarthy et al. 2006), though most were hotter than $\sim 7000 \mathrm{~K}$ because of biases in the SDSS spectroscopic target selection process. The observational efforts of Kilic et al. (2006, 2010) led to spectroscopic confirmation of more than $100 \mathrm{cool}$ WDs, where target selection was based on photometry and proper motions contained in the SDSS DR7 database (Abazajian et al. 2009)

In this work, we utilize data from a suite of surveys and catalogs, including SDSS, the Two Micron All Sky Survey (2MASS; Skrutskie et al. 2006), USNO-B1.0 (Monet et al. 2003), and the proper motion survey of Lépine \& Shara (2005) to identify new WD candidates. From these data, we prioritized targets for follow-up spectroscopy, with emphasis on cool, nearby WDs as well as hotter, and potentially variable (i.e., ZZ Ceti) WDs. We present spectra and physical parameters, derived from spectral energy distribution (SED) and model atmosphere analyses, for 25 newly discovered WDs and one recovered WD (see 4.5). In addition, we present physical parameters for 28 new WDs that have been spectroscopically confirmed by SDSS DR7 spectra but are unpublished elsewhere.

\section{TARGET SELECTION \\ 2.1. Methodology}

Target selection was based on a combination of constraints applied to optical and near-IR photometry, proper motion, and model-adherence. Two independent target selection passes were employed due to high contamination rates experienced with the initial approach. We first queried the SDSS DR7 database for objects with the following stipulations: proper motion $>100$ mas $\mathrm{yr}^{-1}, g$ magnitude $<19.5$, and declination $<+30^{\circ}$. The declination constraint was used to ensure all tar- 
gets could be observed from Cerro Tololo Inter-American Observatory (CTIO), where the first round of spectroscopic observations was taken. Munn et al. (2004) have joined the USNO-B1.0 Catalog with SDSS astrometry (available in the propermotions table of the SDSS database), which allowed us to query for high proper motion objects directly from SDSS. In a second pass, we began with proper motion objects from the catalog of Lépine \& Shara (2005) containing objects with proper

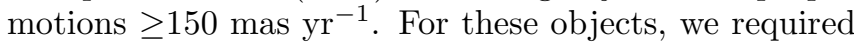
a match in SDSS and employed the $g$ magnitude $<19.5$ limit with no declination constraint. In both approaches, candidate objects were required to have near-IR photometry from 2MASS. Cross-matching between catalogs was achieved using the table matching routines of TOPCAT 6 (Taylor 2005).

The technique of using reduced proper motion (RPM) to identify WDs has been used for decades (e.g., Jones 1972). Conceptually, RPM is used as a proxy for absolute magnitude. The two quantities are connected by the inclusion of tangential velocity. Thus, proper motion coupled with color and apparent magnitude serve to separate generally blue, lower luminosity, and larger tangential velocity WDs from low-metallicity halo subdwarfs (SDs) and main-sequence stars.

Reduced proper motion is defined as

$$
H_{m}=m+5 \log \mu+5=M+5 \log V_{\text {tan }}-3.379
$$

where $m$ is apparent magnitude, $M$ is absolute magnitude, $\mu$ is proper motion in arcseconds $\mathrm{yr}^{-1}$, and $V_{\mathrm{tan}}$ is tangental velocity in $\mathrm{km} \mathrm{s}^{-1}$. In this study, only targets satisfying $H_{g}>15.136+2.727(g-i)$ were kept as WD candidates, a reduced proper motion diagram cut defined by Kilic et al. (2006) that eliminates most of the low-metallicity SDs and virtually all main-sequence stars. A photometric color cut $J-K_{S}<0.5$ was employed to better constrain initially large samples of WD candidates. This color cut was adopted from Subasavage et al. (2008) where they found this to be a clear delimiter between WDs and SD contaminants. All candidates were verified to have noticeable proper motions. These by-eye verifications were carried out by blinking between digitized POSS I and POSS II epochs for each target using the Aladin interactive sky atlas 7 (Bonnarel et al. 2000).

One final model-adherence step was implemented to better probe the cooler regime $\left(T_{\text {eff }}<7000 \mathrm{~K}\right)$ that is plagued by subdwarf contaminants. Remaining targets were crudely compared with WD synthetic photometry models to estimate $T_{\text {eff }}$ values and distances prior to observations. Targets poorly represented by the models as defined below were discarded. We used pure-hydrogen atmospheric WD model 8 and assumed $\log g=8.0$. The model grid was cubic spline interpolated to give a temperature resolution of $10 \mathrm{~K}$. Both modeled and candidate photometry were converted to $\mathrm{F}_{\lambda}$ flux values by the prescription of Holberg \& Bergeron (2006). The resulting SEDs were normalized by the $r$ band flux value. A best fit was determined using a chi-square minimization between model and target normalized flux values. If the photometric error was greater than $0.1 \mathrm{mag}$ then that

\footnotetext{
6 http://www.star.bristol.ac.uk/ mbt/topcat/

7 http://aladin.u-strasbg.fr/

8 http://www .astro.umontreal.ca/ bergeron/CoolingModels/
}

passband was ignored for fitting purposes - this only occurred in the near-IR passbands as the SDSS magnitudes are significantly better down to our adopted magnitude limit of $g=19.5$. A $p$ value for goodness-of-fit was determined using common chi-square lookup tables. We elected to keep only WD candidates with $p>0.95$.

The fitting process provided estimates of $T_{\text {eff }}$ and distance for each target in the final sample. After spectroscopic observations, all bona fide WDs were modeled in a more robust fashion for physical parameter determinations (described in 4.1 and presented here) and should not be confused with this pre-observation model fitting. Finally, all previously identified objects were discarded from our target list, the vast majority of these were published WDs.

\subsection{Completeness Estimates}

We estimate our target-selection completeness by looking at the recovered fraction of SDSS-E06 WDs that met our criteria for both samples (Pass 1 and 2). For a homogeneous comparison, all of the relevant DR8 data (i.e., RA, Dec., proper motion, ugriz, $J H K_{S}$ ) were extracted for the SDSS-E06 sample by matching plate/fiber/mjd designations. A total of 39 objects out of 9316 in SDSSE06 were not recovered because the spectra are not included in the DR8 database (hence, no plate/fiber/mjd designation). We confirm that they are available in the DR7 database. Nevertheless, these few missing objects should not significantly affect the completion statistics.

To compare the SDSS-E06 sample (with DR8 data) to our Pass 1 sample, we implemented identical observational criteria (i.e., proper motion $>100$ mas $\mathrm{yr}^{-1}$, $g<19.5$, Decl. $\left.<+30^{\circ}, J-K_{S}<0.5\right)$ to the SDSSE06 sample. Our Pass 1 sample was then positionally cross-matched to this sample and 19 out of 21 were recovered, implying a completeness of $90 \%$. To estimate the completeness of our Pass 2 sample, the SDSS-E06 sample (with DR8 data) was positionally cross-matched to the LSPM catalog. For all common objects, identical observational criteria (i.e., $g<19.5, J-K_{S}<0.5$ ) as implemented in our Pass 2 were applied. Our Pass 2 sample was compared via LSPM name to the remaining objects once these criteria were applied and 31 out of 35 were recovered, implying a completeness of $89 \%$. Note that in both cases, our adopted limiting magnitude excluded the vast majority of WDs in the SDSS-E06 catalog thereby leaving us with fairly small numbers with which to estimate completeness.

To better understand how our selection criteria affects our completeness, the cuts relating to direct observables (i.e., proper motion, magnitude, color, and declination) were performed first. These samples represent the $100 \%$ complete subsample of SDSS-E06 relevant for each of our passes. We then implement the RPM cut and, in both cases, recover $100 \%$ of the subsample. It was only in the model-adherence cut that recovery rates fell below $100 \%$. Because only a total of 5 unique WDs were not recovered (one unrecovered object belonged to both samples), we looked more carefully at each one to ensure our modeladherence criteria were robust. WD $0756+437$ is a magnetic DA with a field strength of $~ 300 \mathrm{MG}$ (Külebi et al. 2009). WD $1156+132$ is classified as DQpec with deep swan-band carbon absorption. WD1311+129 is classified as a DBA and has the largest hydrogen abundance of the 
Bergeron et al. (2011) sample of DBAs $[\log (\mathrm{H} / \mathrm{He})=-$ 2.90]. The remaining two WDs, WD $1559+534$ and SDSS J101436.01+422622.0 appear to be normal DA white dwarfs that only marginally failed our model-adherence criteria with $p>0.9$. It stands to reason that the first three would fail our model-adherence criteria because their SEDs are not well-represented by a pure- $\mathrm{H}$ model atmosphere. Thus, while our completeness suffered because of our model-adherence criteria, the survey was better able to successfully probe the parameter spaces dominated by contaminants $\left(T_{\text {eff }}<7000 \mathrm{~K}\right)$ to identify nearby WDs as discussed in 4.4 .

\section{DATA AND OBSERVATIONS}

\subsection{Astrometry and Nomenclature}

For new discoveries, we determine WD names in the conventional manner (fully described in Subasavage et al. 2007) by using the target's epoch 1950 equinox 1950 coordinates.

As stated previously, proper motions were initially extracted from the SDSS DR7 database and consisted of combined USNO-B1.0 plus SDSS astrometry (Munn et al. 2004). Munn et al. (2004) demonstrate that the proper motions derived from combined astrometry improves those contained within the USNO-B1.0 catalog by $\sim 25 \%$ when compared to bright, non-moving, spectroscopically-confirmed QSOs. However, Kilic et al. (2006) found that high proper motion objects with neighboring sources within $7^{\prime \prime}$ were more likely to have incorrectly measured proper motions. To remove this source of contamination Kilic et al. (2006) discarded objects with neighbors within $7^{\prime \prime}$. We did not implement this criterion to avoid the possibility of missing true WDs.

After an initial night of spectroscopic observations at CTIO (discussed in Section 3.3), we realized 14 SD and main-sequence contaminates with incorrect proper motion values from the SDSS query. By-eye verification confirmed these to be bona fide proper motion objects, but proper motion magnitudes and position angles were erroneous, suggesting mismatches when combining USNOB1.0 and SDSS astrometry. To mitigate this effect, we initiated a second query using the LSPM-North Catalog (Lépine \& Shara 2005) as a starting point for proper motion values and cross-matched with the SDSS DR7 and 2MASS databases. LSPM-North objects were not initially verified by eye for proper motion confirmation as this was painstakingly done by the original authors. This lead to a significant reduction in contaminants.

Following observations, proper motions for all spectroscopic confirmations (WDs and contaminants) were double checked using the SuperCOSMOS Sky Survey (SSS; Hambly et al. 2001). In cases where the USNOB1.0+SDSS and SSS proper motions were discrepant, a by-eye inspection was performed to confirm position angle. For these objects, we adopt the proper motions extracted from the SSS.

WD names, alternate names (LSPM-North where available and SDSS otherwise), epoch 2000 coordinates and adopted proper motions are listed in Table 1 for both WDs (top) and contaminants (less the WD name, bottom).

\subsection{Photometry}

The psfMag values in the optical ugriz passbands were extracted from the SDSS DR8. The target selections were performed using DR7 photometry but in the interim, DR8 was released and thus, we use these values for SED modeling discussed in Section 4.1. We list these values and their corresponding errors in Table 1.

As a requirement, all candidate WDs had to contain $J H K_{S}$ data in 2MASS. Given that the majority of these targets are near the faint limit of 2MASS, we utilized the UKIRT Infrared Deep Sky Survey (UKIDSS) DR6 Large Area Survey to supplement $J H K_{S}$ for new discoveries where available. The UKIDSS project is defined in Lawrence et al. (2007). UKIDSS uses the UKIRT Wide Field Camera (WFCAM; Casali et al. 2007). The photometric system is described in Hewett et al. (2006), and the calibration is described in Hodgkin et al. (2009). The pipeline processing and science archive are described in Irwin et al. (2009, in preparation) and Hambly et al. (2008). UKIDSS magnitudes were transformed to the 2MASS system using the prescription of Hodgkin et al. (2009). The $J H K_{S}$ values and corresponding errors are listed in Table 1. Converted UKIDSS photometry is listed whenever errors in $J H K_{S}$ are less than 0.05. Otherwise, the values are from 2MASS.

\subsection{Spectroscopy}

Prior to observations, 28 previously unidentified objects had spectra in the SDSS DR7 database confirming their WD nature. These objects are noted in Table 1, but we do not include their spectra as they are freely available in the SDSS database.

Spectroscopic observations were conducted throughout 2010 and early 2011 from both CTIO and Apache Point Observatory (APO). At CTIO the $4 \mathrm{~m}$ Blanco Telescope and Ritchey-Chrétien Spectrograph were used. We selected the KPGL3 grating that covered a wavelength range of 3600-7000 $\mathrm{\AA}$. Observations in 2010 were taken using a 2 !'0 slit width, oriented due north-south, to provide spectral resolution of $6 \AA$. Observations in 2011 were taken using a 4 .' 0 slit width to minimize light loss from differential refraction as the slit was not rotated to the parallactic angle but rather was kept fixed again due north-south. With this configuration, the resolution degraded slightly to $8 \AA$. As can be seen in Figure 1. which contain spectra from both runs, differential refraction was not a significant problem during the first run, nor was the loss of resolution during the second run detrimental for the purpose of spectral classification.

At APO, the Dual Imaging Spectrograph (DIS) on the ARC 3.5m telescope was used with the B400+R300 gratings for spectroscopic follow-up. We achieved $6 \AA$ resolution spanning 3350-9260 , but fringing became problematic beyond $\sim 7000 \AA$. Red and Blue spectroscopic channels were reduced independently. Red channel flux near the dichroic at approximately $5400 \AA$ was found to be highly variable, so wavelengths less than $6300 \AA$ were omitted in the red spectral channel. The default slit width of 1 .' 5 was used for the DIS spectrograph.

During all of the spectroscopic observing runs, flux standards were observed each night for flux calibration and HeNeAr lamps were taken at each telescope pointing for wavelength calibration. Two spectra of each target were obtained to permit cosmic ray rejection. Data were 
reduced using standard IRAF9 routines.

We confirm 19 new WDs from CTIO 4m observations. Figure 1 presents 14 DA discoveries and one DA recovery (see Section 4.5). Figure 2 displays one DQ and two DC WDs. Figure 3 shows spectra and model fits for two DAZ WDs with detectable Ca II absorption features at 3933 and $3968 \AA$. From CTIO, 18 contaminants were observed and their spectra are plotted in Figure 4. Defining absorption features in these contaminant spectra are due to metal and molecular content ( $\mathrm{Ca} \mathrm{II}, \mathrm{CH}, \mathrm{MgH}$ ).

We confirm 6 new WDs from APO $3.5 \mathrm{~m}$ observations: three DA, two DC and one DZ with Ca II absorption in an otherwise featureless spectrum. The upper panels of Figure 5, show these discoveries. We experienced only two contaminants (both likely SDs) and their spectra are plotted in the lower two panels of Figure 5

\section{ANALYSIS}

\subsection{Modeling of Physical Parameters}

Our model atmospheres for WDs are similar to those described at length in Liebert et al. (2005, and references therein) and Bergeron et al. (1995a), with several improvements discussed in Tremblay \& Bergeron (2007) and Tremblay et al. (2011). In particular, we now make use of the improved Stark broadening profiles for the hydrogen lines developed by Tremblay \& Bergeron (2009). Our models for DQ and DZ stars, which include metals and molecules in the equation of state and opacity calculations, are described in detail in Dufour et al. (2005) and Dufour et al. (2007), respectively.

Table 2 contains SED-derived $T_{\text {eff }}$ (column 2) and distance (column 4) for WDs with pure $\mathrm{H}$ or pure He atmospheres (denoted in column 3). A complete discussion of our SED-fitting procedure can be found in Bergeron et al. (2001). For "polluted" WDs (DAZ, DQ, DZ), these parameters rely on the iterative, combined SED and spectral fitting procedures defined in Dufour et al. (2005, 2007). Metal abundances derived from the model fits for these targets are listed in the notes section of Table 2. For all cases, we assume $\log g=$ 8.0. Spectral subtypes (column 5) are determined for the DA WDs using the temperature index of McCook \& Sion (1999), where the temperature index equals $50,400 / T_{\text {eff }}$ - In addition, spectroscopic line profile fitting was performed as described in Bergeron et al. (1992) for all DAs (and one DBA - WD 1457+249) with sufficient line absorption and signal-to-noise to produce a reliable fit. These results are listed in columns 6 and 7 of Table 2 .

\subsection{Metal-Polluted DA White Dwarfs}

Figure 3 displays spectra and model fits for WD $0920+012$ and WD 1408+029. These two DA WDs exhibit $\mathrm{Ca}$ II $\mathrm{H} \& \mathrm{~K}$ features and earn the classification of DAZ. Hypotheses to explain these spectral features include enrichment from (1) the interstellar medium or, (2) debris disk accretion, with the latter being heavily favored (Farihi et al. 2010).

WD $0920+012$ is estimated to be at $33.4 \mathrm{pc}$, while WD $1408+029$ is estimated to be at $26.5 \mathrm{pc}$, and both

9 IRAF is distributed by the National Optical Astronomy Observatry, which is operated by the Association of Universities for Research in Astronomy, Inc., under cooperative agreement with the National Science Foundation are modeled to have a $\log (\mathrm{Ca}) /(\mathrm{H})=-9.0$. Given their relatively bright apparent magnitudes $(g \sim 17)$, these targets would be excellent candidates for follow-up IR studies to possibly detect emission from the accretion disk and better characterize the system.

\subsection{ZZ Ceti White Dwarfs}

Two of our confirmed WDs were modeled to lie in the ZZ Ceti instability strip: WD $1419+062$ and WD $2102+233$. Figure 6 shows the instability strip as recently redefined by Gianninas et al. (2011) using improved model spectra with the new Stark profiles described above.

Differential photometry was performed on both $\mathrm{ZZ}$ Ceti candidates. Candidate flux was normalized by bright non-varying stars in the same field to obtain a differential light curve. The light curve was then normalized by the mean value to obtain fractional variation about the mean. Frequency content was analyzed using the magnitude of the Fourier Transform (FT) of the time series data.

WD $2102+233$ was observed using the CTIO $0.9 \mathrm{~m}$ telescope using the full 13! 6 field and the BG 40 filter. The time cadence was $\sim 50$ seconds. As can be seen in Figure 7. we identify a dominant pulsational period at $\sim 800$ seconds with an amplitude of $\sim 2.6 \%$.

WD 1419+062 was observed using the CTIO 1.0m telescope, with a 20 '0 field, and the BG 40 filter. We detect no obvious pulsations from these observations (see Figure 8). From the FT, the observed noise level is $\sim 0.25 \%$, with no obvious peaks. With our less-than-optimal sampling rate ( $\sim 100$ seconds), we could only hope to detect periods $>200$ seconds based on the Nyquist sampling theorem, so this object may be pulsating outside our detection sensitivity. However, considering its location in Figure 6] lying near the red edge of the instability strip, we would expect a longer period variable.

\subsection{Nearby White Dwarfs}

We find three new WDs with distance estimates within the 25 pc horizon of interest (WD $1338+052$ at $13.7 \pm 2.7$ pc, WD $1630+089$ at $13.2 \pm 2.3 \mathrm{pc}$, and WD $2119+040$ at $22.1 \pm 3.6 \mathrm{pc}$ ) that has been adopted from the Catalog of Nearby Stars (CNS; Gliese \& Jahreiß 1991) and the NStars Database (Henry et al. 2003). Holberg et al. (2008) determine that the 20 pc WD sample is $~ 80 \%$ complete based on the assumption that the $13 \mathrm{pc}$ WD sample is complete. Two of these WDs, and possibly the third if it is slightly less distant than expected, will lie within $20 \mathrm{pc}$ if proximity is confirmed. Moreover, WD $1338+052$ and WD $1630+089$ are modeled to lie at a distance very near to $13 \mathrm{pc}$. If either object proves to lie within $13 \mathrm{pc}$, the local WD population must be denser than previously thought. With any new WD discoveries within $13 \mathrm{pc}$, a constant-density extrapolation would increase the amount of missing WDs in the 13 pc to 20 pc range. The exact current completeness statistics will depend on more robust distance determinations.

These three objects are being observed for trigonometric parallaxes via the Cerro Tololo Inter-American Observatory Parallax Investigation (CTIOPI; Jao et al. 2005; Henry et al. 2006; Subasavage et al. 2009; Riedel et al. 2010; Jao et al. 2011) program to confirm proximity. To 
the best of our knowledge, the two WDs estimated to be within 20pc are the nearest WDs discovered using SDSS data, if trigonometric parallaxes confirm proximity. Continuing discoveries of the coolest WDs, especially those nearby and thus suitable for parallax measurements, will provide anchor points for WD atmospheric models that are vital to widely-used cosmic dating techniques.

\subsection{Notes in Individual Systems}

WD 0351-002 is also known as SA 95-42 and is a popular spectrophotometric standard star. Oke (1990) lists this object along with 24 other spectrophotometric standard candidates, including spectra. For reasons unclear, this is one of three objects that do not have a spectral type in Table 1 of Oke (1990) yet the spectrum published in that same work shows broad Balmer absorption indicative of a DA WD. We include it here as a new discovery.

WD $\mathbf{0 4 1 2 + 0 6 5}$ is also known as GD 59. It was classified as a WD suspect by Giclas et al. (1965), but we found no spectroscopic confirmation in the literature even though it is listed as a WD in Simbad. We include it here as a new discovery.

WD $\mathbf{1 4 0 2 + 0 6 5}$ is also known as PG $1402+065$ and was previously labeled as a subdwarf in the catalogs of Green et al. (1986) and subsequently included in the subdwarf catalog of Kilkenny et al. (1988), based on the previous determination. Our spectrum (see Figure11) clearly shows broad Balmer absorption and thus, we include it here as a new WD discovery. In fact, this WD is the hottest of those spectroscopically observed from CTIO plotted in Figure 1 $\left(T_{\text {eff }}=26,190 \mathrm{~K}\right)$.

WD $\mathbf{1 4 1 9 + 0 6 2}$ is also known as PG $1419+062$ and was first published by Green (1980) as a DA. However, it appears in Green et al. (1986) with a "sd" designation indicative of being a hot subdwarf. It then appears in the catalog of Kilkenny et al. (1988), again classified as a hot subdwarf based on the Green et al. (1986) designation. Here we confirm it to be a bona fide DA WD and suspect the Green et al. (1986) designation is a typo. We include the spectroscopy and the modeled parameters yet do not classify this object as a new discovery. This object is also discussed in Section 4.3 as a ZZ Ceti candidate.

WD $\mathbf{1 4 3 4 + 1 5 9}$ is also known as GD 168. It was classified as a WD suspect by Giclas et al. (1965), but we found no spectroscopic confirmation in the literature even though it is listed as a WD in Simbad. We include it here as a new discovery.

WD $1457+249$ has a spectrum dominated by He and was SED-modeled using a pure He model atmosphere. During the spectroscopic line fitting analysis, it became evident that, because of weak Balmer $\mathrm{H} \beta$ absorption, trace amounts of $\mathrm{H}$ existed in the atmosphere. Thus, this object is classified as a DBA with the best-fitting model including $\log (\mathrm{H} / \mathrm{He})=-5.8 \pm 0.1$.

\section{DISCUSSION}

We present and characterize 54 WDs. Of these, 26 objects were spectroscopically observed from either CTIO or APO (including recovered known object WD $1419+062$, as discussed in Section 4.5), while the remaining 28 objects have spectra available in the SDSS DR7 database for a total of 53 new WDs. It is likely the SDSS DR7 spectra will be contained in the forthcoming DR7
White Dwarf Catalog described by Kleinman (2010). Additionally, 21 objects overlap (17 from SDSS spectra and 4 photometrically-selected) between this study and a recent publication by Girven et al. (2011) to identify DA WDs in SDSS.

We find three WDs expected to be in the local neighborhood, with the closest (WD1630+089) expected to be $13.2 \mathrm{pc}$ distant. Twenty-one WDs are modeled to have $T_{\text {eff }}<7000 \mathrm{~K}$, with four of those objects modeled to have $T_{\text {eff }}<5000 \mathrm{~K}$. We also confirm WD $2102+233$ as a new ZZ Ceti star.

Our survey is summarized in Table 3. Briefly, our selection criteria were designed to optimally identify cooler WDs in parameter spaces where contaminants dominate. As such, we realized some contamination in our final sample, both identified by our spectra and also from the literature (Table 3 column 10) as listed by Simbad. Our methodology was initially impacted by problematic proper motions leading to significant contamination by subdwarfs and main-sequence stars (Pass 1 in Table 3). Once correct proper motions are applied to these objects (see Figure 9), it is clear the contamination would have been largely avoided as these objects fall above our RPM cut. Our corrected contamination is generally consistent with Kilic et al. (2010) who show decreasing contamination rates with increasing $V_{\mathrm{tan}}$ cutoffs. They quote a $1.3 \%$ contamination rate for $V_{\tan } \geq 30 \mathrm{~km} \mathrm{~s}^{-1}$ and indeed, we find one contaminant scattered within the $V_{\tan } \geq 30 \mathrm{~km}$ $\mathrm{s}^{-1}$ cutoff. While we show yet another example of the effectiveness of RPM to isolate WDs, there is an inherent bias against the slowest moving WDs. It is unlikely that this bias will be completely removed until a magnitudelimited astrometric survey is conducted, such as Gaia or LSST.

Only a subset of our WD candidates were able to be observed because of telescope time constraints. The last column in Table 3 shows the number of targets left unobserved. Furthermore, all of our targets were constrained to the SDSS footprint thereby covering only a fraction of the sky (Table 3 column 3 ) that is largely weighted towards northern declinations. For these reasons, we expect that more WDs of interest remain undiscovered. Highly anticipated photometric surveys such as Pan-STARRS and the Dark Energy Survey (DES) will certainly aid in the discovery of additional WDs. With this in mind, we publish our observed contaminates to add to those already known to have similar photometric properties and proper motions to WDs (e.g., Kilic et al. 2006, 2010). By identifying large samples of contaminants now, disentangling them from WD candidates may be easier in the future using empirical and statistical methods. Newling et al. (2011) provide an example of statistical techniques based on photometry to classify supernovae. For similar techniques to work in our context, large robust training sets of both SD and WD exemplars are needed before any statistical assertions can be made, and applied to large data sets.

We are grateful to the anonymous referee, who provided detailed comments and suggestions that enabled the manuscript to be more concise and clear. C.S. and J.P.S. wish to thank the 2010 CTIO REU Program, of which C.S. was a student (the majority of this work was 
conducted during the program under the direction of J.P.S.). In particular, we thank Chris Smith and Nicole van der Bliek for director's discretionary time on the $4 \mathrm{~m}$ Blanco Telescope at CTIO and Suzanne Hawley for director's discretionary time on the APO ARC $3.5 \mathrm{~m}$ telescope. We thank Bart Dunlap for spectral analysis tips, and Adam Kowalski for DIS reduction advice.

This work was, in part, based on observations obtained with the Apache Point Observatory 3.5-meter telescope, which is owned and operated by the Astrophysical Research Consortium. This work is supported in part by the NSERC Canada and by the Fund FQRNT (Québec). $\mathrm{PD}$ is a CRAQ postdoctoral fellow.

This publication makes use of data products from the Two Micron All Sky Survey, which is a joint project of the University of Massachusetts and the Infrared Processing and Analysis Center/California Institute of Technology, funded by the National Aeronautics and Space Administration and the National Science Foundation.

Funding for the SDSS and SDSS-II has been provided by the Alfred P. Sloan Foundation, the Participating Institutions, the National Science Foundation, the U.S. Department of Energy, the National Aeronautics and Space Administration, the Japanese Monbukagakusho, the Max Planck Society, and the Higher Education Funding Council for England. The SDSS Web Site is http://www.sdss.org/. The SDSS is managed by the Astrophysical Research Consortium for the Participating Institutions. The Participating Institutions are the American Museum of Natural History, Astrophysical Institute Potsdam, University of Basel, University of Cambridge, Case Western Reserve University, University of Chicago, Drexel University, Fermilab, the Institute for Advanced Study, the Japan Participation Group, Johns Hopkins University, the Joint Institute for Nuclear Astrophysics, the Kavli Institute for Particle Astrophysics and Cosmology, the Korean Scientist Group, the Chinese Academy of Sciences (LAMOST), Los Alamos National Laboratory, the Max-Planck-Institute for Astronomy (MPIA), the Max-Planck-Institute for Astrophysics (MPA), New Mexico State University, Ohio State University, University of Pittsburgh, University of Portsmouth, Princeton University, the United States Naval Observatory, and the University of Washington.

\section{REFERENCES}

Abazajian, K. N., et al. 2009, ApJS, 182, 543

Aihara, H., et al. 2011, ApJS, 193, 29

Adelman-McCarthy, J. K., et al. 2006, ApJS, 162, 38

Bergeron, P., Saffer, R. A., \& Liebert, J. 1992, ApJ, 394, 228

Bergeron, P., Saumon, D., \& Wesemael, F. 1995a, ApJ, 443, 764

Bergeron, P., Leggett, S. K., \& Ruiz, M. T. 2001, ApJS, 133, 413

Bergeron, P., Wesemael, F., Dufour, P., et al. 2011, ApJ, 737, 28

Bonnarel, F., et al. 2000, A\&AS, 143, 33

Casali, M., et al. 2007, A\&A, 467, 777

Dufour, P., Bergeron, P., \& Fontaine, G. 2005, ApJ, 627, 404

Dufour, P., et al. 2007, ApJ, 663, 1291

Eisenstein, D. J., et al. 2006, ApJS, 167, 40

Farihi, J., Jura, M., Lee, J.-E., \& Zuckerman, B. 2010, ApJ, 714, 1386

Gianninas, A., Bergeron, P., \& Ruiz, M. T. 2011, ApJ, 743, 138

Giclas, H. L., Burnham, R., \& Thomas, N. G. 1965, Lowell Observatory Bulletin, 6, 155

Girven, J., Gänsicke, B. T., Steeghs, D., \& Koester, D. 2011, MNRAS, 417, 1210

Gliese, W., \& Jahreiß, H. 1991, On: The Astronomical Data Center CD-ROM: Selected Astronomical Catalogs, Vol. I; L.E. Brotzmann, S.E. Gesser (eds.), NASA/Astronomical Data Center, Goddard Space Flight Center, Greenbelt, MD,

Green, R. F. 1980, ApJ, 238, 685

Green, R. F., Schmidt, M., \& Liebert, J. 1986, ApJS, 61, 305

Hambly, N. C., Davenhall, A. C., Irwin, M. J., \& MacGillivray, H. T. 2001, MNRAS, 326, 1315

Hambly, N. C., et al. 2008, MNRAS, 384, 637

Henry, T. J., Backman, D. E., Blackwell, J., Okimura, T., \& Jue, S. 2003, Astrophysics and Space Science Library, 289, 111

Henry, T. J., Jao, W.-C., Subasavage, J. P., Beaulieu, T. D., Ianna, P. A., Costa, E., \& Méndez, R. A. 2006, AJ, 132, 2360

Hewett, P. C., Warren, S. J., Leggett, S. K., \& Hodgkin, S. T. 2006, MNRAS, 367, 454

Hodgkin, S. T., Irwin, M. J., Hewett, P. C., \& Warren, S. J. 2009, MNRAS, 394, 675

Holberg, J. B., \& Bergeron, P. 2006, AJ, 132, 1221
Holberg, J. B., et al. 2008, AJ, 135, 1225

Jao, W.-C., Henry, T. J., Subasavage, J. P., Brown, M. A., Ianna, P. A., Bartlett, J. L., Costa, E., \& Méndez, R. A. 2005, AJ, 129, 1954

Jao, W.-C., Henry, T. J., Subasavage, J. P., Winters, J. G., Riedel, A. R., \& Ianna, P. A. 2011, AJ, 141, 117

Jones, E. M. 1972, ApJ, 177, 245

Kilic, M., et al. 2006, AJ, 131, 582

Kilic, M., et al. 2010, ApJS, 190, 77

Kilkenny, D., Heber, U., \& Drilling, J. S. 1988, South African Astronomical Observatory Circular, 12, 1

Kleinman, S. J. 2010, American Institute of Physics Conference Series, 1273, 156

Külebi, B., Jordan, S., Euchner, F., Gänsicke, B. T., \& Hirsch, H. 2009, A\&A, 506, 1341

Lawrence, A., et al. 2007, MNRAS, 379, 1599

Leggett, S. K., Ruiz, M. T., \& Bergeron, P. 1998, ApJ, 497, 294

Lépine, S., \& Shara, M. M. 2005, AJ, 129, 1483

Liebert, J., Bergeron, P., \& Holberg, J. B. 2005, ApJS, 156, 47

McCook, G. P., \& Sion, E. M. 1999, ApJS, 121, 1

Monet, D. G., et al. 2003, AJ, 125, 984

Munn, J. A., et al. 2004, AJ, 127, 3034

Newling, J., et al. 2011, MNRAS, 545

Oke, J. B. 1990, AJ, 99, 1621

Riedel, A. R., et al. 2010, AJ, 140, 897

Skrutskie, M. F., et al. 2006, AJ, 131, 1163

Subasavage, J. P., et al. 2007, AJ, 134, 252

Subasavage, J. P., et al. 2008, AJ, 136, 899

Subasavage, J. P., et al. 2009, AJ, 137, 4547

Taylor, M. B. 2005, Astronomical Data Analysis Software and Systems XIV, 347, 29

Tremblay, P.-E., \& Bergeron, P. 2007, ApJ, 657, 1013

Tremblay, P.-E., \& Bergeron, P. 2009, ApJ, 696, 1755

Tremblay, P.-E., Bergeron, P., \& Gianninas, A. 2011, ApJ, 730, 128

York, D. G., et al. 2000, AJ, 120, 1579 
Table 1. Astrometry and Photometry

\begin{tabular}{|c|c|c|c|c|c|c|c|c|c|c|c|c|c|c|c|c|c|c|c|c|c|c|}
\hline $\begin{array}{c}\text { WD } \\
\text { Name } \\
\end{array}$ & $\begin{array}{c}\text { Alternate } \\
\text { Name }\end{array}$ & R.A. $\quad(J 2000.0)$ & 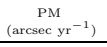 & $\begin{array}{l}\begin{array}{l}\text { P.A. } \\
\text { (deg) }\end{array} \\
\end{array}$ & $\begin{array}{l}\text { PM } \\
\text { Ref. } \\
\end{array}$ & $u$ & $\sigma_{u}$ & $g$ & & & $\sigma_{r}$ & $i$ & $\sigma_{i}$ & $z$ & $\sigma_{z}$ & J & $\sigma_{J}$ & ${ }_{H}^{H}$ & $\sigma_{H}$ & $K_{s}$ & $\sigma_{K_{s}}$ & Source \\
\hline \multicolumn{23}{|c|}{ Spectroscopically Confrmed White Dwarfs } \\
\hline & & & & & & & & & & & & & & & & & & & & & & \\
\hline & & & & & & & & & & & & & & & & & & & & & & \\
\hline 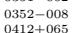 & 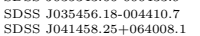 & $\begin{array}{l}035456.16-004410.5 \\
041458.22 \\
0406408.9\end{array}$ & 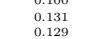 & 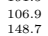 & $\begin{array}{l}\text { SDS } \\
\text { SDS }\end{array}$ & $\begin{array}{l}18.0940 \\
11.701 \\
150\end{array}$ & $\begin{array}{l}0.016 \\
0.05\end{array}$ & $\begin{array}{l}17.364 \\
15.256\end{array}$ & 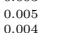 & $\begin{array}{l}16.994 \\
1.550\end{array}$ & $\begin{array}{l}0.005 \\
0.004\end{array}$ & 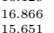 & 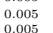 & $\begin{array}{l}16.833 \\
11.591 \\
1.591\end{array}$ & $\begin{array}{l}30.010 \\
1 \\
1007\end{array}$ & 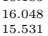 & 108 & $\begin{array}{l}5.858 \\
5.556\end{array}$ & o11 011 & $\begin{array}{l}0.740 \\
5.737\end{array}$ & 0.022 & $\begin{array}{l}\text { CTIO } \\
\text { CTIO }\end{array}$ \\
\hline $\begin{array}{l}+250 \\
+2543 \\
+243\end{array}$ & 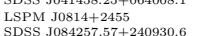 & 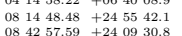 & 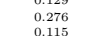 & $\begin{array}{l}240.8 \\
2448 \\
248\end{array}$ & $\begin{array}{l}\text { LEP } \\
\text { SDS }\end{array}$ & $\begin{array}{l}17.1880 \\
17.361 \\
100\end{array}$ & $\begin{array}{l}0.009 \\
0.017 \\
0.017\end{array}$ & $\begin{array}{l}16.962 \\
176928 \\
17428\end{array}$ & $\begin{array}{l}0.09 \\
0.005 \\
0.05\end{array}$ & 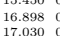 & & 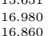 & 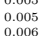 & 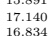 & & $\begin{array}{l}10.670 \\
11.670 \\
1.988\end{array}$ & & $\begin{array}{l}16.400 \\
15.99 \\
1597\end{array}$ & & $\begin{array}{l}17.280 \\
17.602\end{array}$ & $\begin{array}{l}\text { Null } \\
\text { Nula } \\
0.192\end{array}$ & 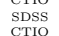 \\
\hline $\begin{array}{l}040+243 \\
0851+280\end{array}$ & 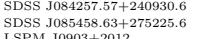 & 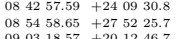 & 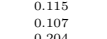 & $\begin{array}{l}242.8 \\
227.7 \\
27.7\end{array}$ & $\begin{array}{l}\text { SDS } \\
\text { SDS } \\
\text { SDP }\end{array}$ & $\begin{array}{l}18.3617 \\
18.327 \\
18\end{array}$ & $\begin{array}{l}0.017 \\
0.017\end{array}$ & $\begin{array}{l}17.48 \\
177709 \\
1709\end{array}$ & $\begin{array}{l}0.005 \\
0.005\end{array}$ & $\begin{array}{l}177030 \\
17.501 \\
\mathrm{~g}\end{array}$ & & $\begin{array}{l}17.860 \\
17.466 \\
7\end{array}$ & 然.006 & $\begin{array}{l}16.734 \\
17.521 \\
\mathrm{l}\end{array}$ & & 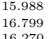 & & 16.9.977 & $\begin{array}{c}20 \\
\text { ull } \\
\text { s. }\end{array}$ & $\begin{array}{l}15.602 \\
16.771\end{array}$ & $\begin{array}{l}0.192 \\
\text { Null } \\
\text { Nul }\end{array}$ & $\begin{array}{l}\text { CrTI } \\
\text { SDSS } \\
\text { sSS }\end{array}$ \\
\hline og & $\begin{array}{l}\text { LLPM Jo903+2012 } \\
\text { LSPM J0910+2156 }\end{array}$ & 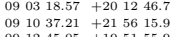 & $\begin{array}{l}0.204 \\
0.301 \\
0\end{array}$ & $\begin{array}{l}18.5 \\
130.1 \\
\end{array}$ & $\begin{array}{l}\text { LEP } \\
\text { LEP }\end{array}$ & $\begin{array}{l}18.588 \\
18.675 \\
0\end{array}$ & $\begin{array}{l}0.011 \\
0.020\end{array}$ & 177.671 & & 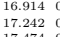 & & & $\begin{array}{l}0.005 \\
0.017\end{array}$ & 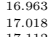 & & & & & & 16.860 & 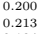 & $\begin{array}{l}\text { SDSS } \\
\text { CTIO }\end{array}$ \\
\hline $\begin{array}{l}9+200 \\
0+012\end{array}$ & PM J0922+0103 & 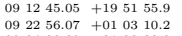 & 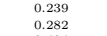 & 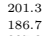 & $\begin{array}{l}\text { LEP } \\
\text { LEP }\end{array}$ & $\begin{array}{l}17.688 \\
17.6640 \\
0\end{array}$ & $\begin{array}{l}0.035 \\
0.020\end{array}$ & 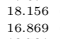 & .0.022 & $\begin{array}{l}17.44 \\
1.699 \\
\end{array}$ & & $\begin{array}{ll}17.188 \\
16.388\end{array}$ & . 0.021 & 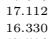 & & 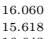 & & 515.898 & 1010 & $\begin{array}{l}15.503 \\
15.309\end{array}$ & $\begin{array}{l}0.184 \\
0.016\end{array}$ & $\begin{array}{l}\text { CrIo } \\
\text { Crio }\end{array}$ \\
\hline $\begin{array}{l}1+315 \\
8+192 \\
8+192\end{array}$ & $\begin{array}{l}\text { LSPM J0924+31200 } \\
\text { LSPM J J951+1900 }\end{array}$ & 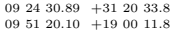 & $\begin{array}{l}0.424 \\
0.274\end{array}$ & 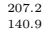 & $\begin{array}{l}\text { LEP } \\
\text { LEP }\end{array}$ & $\begin{array}{l}2.028 \\
18.934 \\
0\end{array}$ & $\begin{array}{l}0.077 \\
0.023\end{array}$ & $\begin{array}{l}18.731 \\
81.14\end{array}$ & 0.0 .099 & $\begin{array}{l}17.944 \\
1.672\end{array}$ & & $\begin{array}{l}17.631 \\
77.541\end{array}$ & $\begin{array}{c}0 \\
0 \\
0\end{array}$ & $\begin{array}{l}17.516 \\
11.552 \\
\mathrm{C}\end{array}$ & & 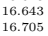 & & 5.9706 & $\begin{array}{l}87 \\
98\end{array}$ & $\begin{array}{l}16.910 \\
17.326\end{array}$ & $\begin{array}{l}\text { Null } \\
\text { Null }\end{array}$ & $\begin{array}{l}\text { SDSS } \\
\text { CTIO }\end{array}$ \\
\hline $3+1$ & PM J1026 + 1439 & $\begin{array}{l}102628.18 \\
1027 \\
1020\end{array}$ & 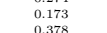 & $\begin{array}{l}237.9 \\
23627-9\end{array}$ & $\begin{array}{l}\text { LEP } \\
\text { LEP }\end{array}$ & 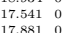 & $\begin{array}{l}0.012 \\
0.012\end{array}$ & 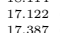 & 0.005 & $\begin{array}{l}17.140 \\
71.720\end{array}$ & & & 006 & 17.344 & & & & 5.524 & & 6.617 & ull & sis \\
\hline 1032+230 & $\begin{array}{l}1027+1928 \\
1034+2245\end{array}$ & $\begin{array}{l}102747.780+192824.6 \\
103443.40+224548.3\end{array}$ & $\begin{array}{l}0.378 \\
0.222 \\
-10\end{array}$ & $\begin{array}{l}263.7 \\
263.5 \\
-2\end{array}$ & $\begin{array}{l}\text { LEP } \\
\text { LEP }\end{array}$ & $\begin{array}{l}17.881 \\
17.376 \\
0\end{array}$ & $\begin{array}{l}0.013 \\
0.010\end{array}$ & $\begin{array}{l}17.38782 \\
16.702\end{array}$ & $\begin{array}{l}0.005 \\
0.005 \\
-10\end{array}$ & $\begin{array}{l}17.228 \\
16.560\end{array}$ & & $\begin{array}{l}17.178 \\
6.643\end{array}$ & $\begin{array}{l}0.005 \\
0.006\end{array}$ & 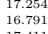 & & $\begin{array}{l}16.4241 \\
16.240\end{array}$ & & $\begin{array}{l}6.441 \\
6.410 \\
6\end{array}$ & & $\begin{array}{l}6.939 \\
5.750\end{array}$ & vull & $\begin{array}{l}\text { SDSS } \\
\text { SDSS }\end{array}$ \\
\hline & $\begin{array}{l}\text { M J J11057+23366 } \\
\text { M J11446 }\end{array}$ & $\begin{array}{l}105630.08+2336186.5 \\
110709.72+1446545\end{array}$ & $\begin{array}{l}0.270 \\
0.278 \\
-10\end{array}$ & $\begin{array}{l}222.2 \\
292.9\end{array}$ & $\begin{array}{l}\text { LEP } \\
\text { LEP }\end{array}$ & $\begin{array}{l}17.919 \\
17.376 \\
0\end{array}$ & & $\begin{array}{l}18.414 \\
16.797\end{array}$ & $\begin{array}{l}0.029 \\
0.018 \\
0\end{array}$ & $\begin{array}{l}17752 \\
16.539 \\
\end{array}$ & & 16.535 & & 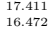 & & & & $\begin{array}{l}6.566 \\
5.470 \\
5\end{array}$ & & $\begin{array}{l}6.246 \\
\text { 5.424 }\end{array}$ & wall & $\begin{array}{l}\text { CTIO } \\
\text { CTIO }\end{array}$ \\
\hline & & $\begin{array}{l}111316.59+285907.8 \\
111858.84+283657.8 \\
118\end{array}$ & 0.0394 & $\begin{array}{l}205.4 \\
279.1\end{array}$ & $\begin{array}{l}\text { LEP } \\
\text { LEP }\end{array}$ & $\begin{array}{l}20.213 \\
210.950 \\
0\end{array}$ & & 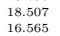 & $\begin{array}{l}0.007 \\
0.004\end{array}$ & $\begin{array}{l}17.734 \\
1.652\end{array}$ & & $\begin{array}{l}17.440 \\
6.586\end{array}$ & 0.007 & 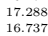 & $\begin{array}{l}0.015 \\
70.099\end{array}$ & & & . & & $\begin{array}{l}15.870 \\
156.170\end{array}$ & $\begin{array}{l}\text { Null } \\
\text { Null }\end{array}$ & $\begin{array}{l}\text { SDSS } \\
\text { SDSS }\end{array}$ \\
\hline $\begin{array}{l}11116-103 \\
111103\end{array}$ & $\begin{array}{l}\text { SDSS J111930.84 } \\
\text { LSPM J1122+28. }\end{array}$ & 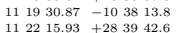 & $\begin{array}{l}0.160 \\
0.276\end{array}$ & $\begin{array}{l}333.6 \\
255.8\end{array}$ & $\begin{array}{l}\text { SDS } \\
\text { LEP }\end{array}$ & $\begin{array}{l}17.159 \mathrm{~g} \\
17.382 \mathrm{~g}\end{array}$ & $\begin{array}{l}0.009 \\
0.003\end{array}$ & 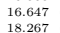 & & $\begin{array}{l}16.493 \\
11.705\end{array}$ & & & $\begin{array}{l}0.005 \\
0.007 \\
0\end{array}$ & $\begin{array}{l}16.509 \\
11.561 \\
1.561\end{array}$ & & $\begin{array}{l}15.903 \\
11.520\end{array}$ & & . 7.762 & & $\begin{array}{l}15.415 \\
16.180\end{array}$ & 0.216 & $\begin{array}{l}\text { CTIO } \\
\text { CDSS } \\
\text { sis }\end{array}$ \\
\hline 1134+20 & $\begin{array}{l}\text { LSPM J11237+204 } \\
\text { LSPS J117 }\end{array}$ & 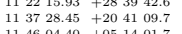 & 0.0367 & 256.5 258 & $\begin{array}{l}\text { LEP } \\
\text { SEP }\end{array}$ & $\begin{array}{l}17.3828 \\
17.931\end{array}$ & & 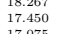 & & 17.357 & & & & & & & & 6.430 & & 6.640 & $\begin{array}{l}\text { Noll } \\
\text { Null }\end{array}$ & $\begin{array}{l}\text { ss } \\
\text { ss }\end{array}$ \\
\hline & 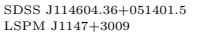 & 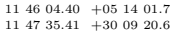 & $\begin{array}{l}0.102 \\
0.183\end{array}$ & $\begin{array}{l}275.6 \\
278.2 \\
2\end{array}$ & $\begin{array}{l}\mathrm{SDS} \\
\mathrm{LEP}\end{array}$ & $\begin{array}{l}17.865 \\
18.361 \\
\mathrm{~g}\end{array}$ & $\begin{array}{l}0.010 \\
0.017\end{array}$ & $\begin{array}{l}17.075 \\
17.822 \\
-10\end{array}$ & $\begin{array}{l}0.004 \\
0.005\end{array}$ & $\begin{array}{l}16.814 \\
17.627\end{array}$ & & $\begin{array}{ll}6.7331 \\
77.602\end{array}$ & 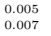 & 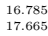 & 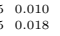 & & & .099 & ${ }_{111}^{15}$ & 6.660 & will & $\begin{array}{l}\text { SDSS } \\
\text { SDSS }\end{array}$ \\
\hline & & 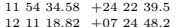 & & & & & & & & & & & & & & & & & & & & \\
\hline 1224+321 & 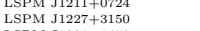 & $\begin{array}{l}1211188.82+072448.2 \\
122724.27+315024.2\end{array}$ & 0.204 & 152.3 & LEP & $\begin{array}{l}17.564 .488 \mathrm{~g} \\
17.48\end{array}$ & $\begin{array}{l}0.023 \\
0.011\end{array}$ & $\begin{array}{l}17.158 \\
16.838\end{array}$ & $\begin{array}{l}0.020 \\
0.004 \\
0.0250\end{array}$ & $\begin{array}{l}16.534 \\
16.578\end{array}$ & $\begin{array}{l}0.004 \\
0.04\end{array}$ & 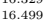 & 0.004 & 16.523 & $\begin{array}{l}80.016 \\
0.009\end{array}$ & $\begin{array}{l}15.388 \\
15.890\end{array}-100$ & $\begin{array}{l}30.055 \\
0.085 \\
3\end{array}$ & 15.505 & 135 & $\begin{array}{l}4.9 .95 \\
5.490\end{array}$ & $\begin{array}{l}0.011 \\
0.214\end{array}$ & SDSS \\
\hline & $\begin{array}{l}\text { LSPM J J12311+1452 } \\
\text { SDSS J124140.03-07305. }\end{array}$ & 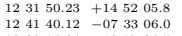 & $\begin{array}{l}0.158 \\
0.174\end{array}$ & $\begin{array}{l}129.9 \\
279.2\end{array}$ & $\begin{array}{l}\text { LEP } \\
\text { SDS }\end{array}$ & $\begin{array}{l}179.844 \mathrm{~g} \\
17.248 \mathrm{~g}\end{array}$ & $\begin{array}{l}0.027 \\
0.010\end{array}$ & 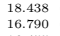 & $\begin{array}{l}0.077 \\
0.004 \\
0.0750\end{array}$ & $\begin{array}{l}17.966 \\
16.869\end{array}$ & $\begin{array}{l}0.007 \\
0.005\end{array}$ & $\begin{array}{l}17.818 \\
16.990\end{array}$ & 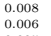 & $\begin{array}{l}177.92 \\
17.137 \\
\mathrm{C}\end{array}$ & $\begin{array}{l}0161 \\
012\end{array}$ & 16.632 16.632 & 2 & $\begin{array}{l}16.768 \\
17.658\end{array}$ & 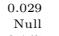 & $\begin{array}{l}16.717 \\
16.284\end{array}$ & $\begin{array}{l}0.052 \\
\text { Null } \\
\text { wats }\end{array}$ & $\begin{array}{l}\text { SDSS } \\
\text { CTIO }\end{array}$ \\
\hline+1 & $\begin{array}{l}\text { SDSS J13001.77 } \\
\text { LSPM J1301+26 }\end{array}$ & 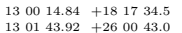 & $\begin{array}{l}0.14 \\
0.18\end{array}$ & 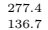 & $\begin{array}{l}\substack{\text { SDS } \\
\text { LEP }} \\
\text { d }\end{array}$ & $\begin{array}{l}18.912 \\
18.317 \\
\mathrm{~g}\end{array}$ & $\begin{array}{l}0.009 \\
0.026\end{array}$ & $\begin{array}{l}17.455 \\
7.747 \\
\end{array}$ & 0.0016 & $\begin{array}{l}17.625 \\
17.554\end{array}$ & & o6 02 & & 17.752 & & 16.926 & & .183 & & 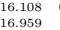 & No.273 & \\
\hline & $\begin{array}{l}\text { SDSS J131023.76+ } \\
\text { LSPM M1324.0857 }\end{array}$ & $\begin{array}{l}131023.77 \\
13240420.5 \\
12436.86\end{array}$ & $\begin{array}{l}0.149 \\
0.219 \\
0\end{array}-19$ & $\begin{array}{l}197.6 \\
153.3 \\
-150\end{array}$ & $\begin{array}{l}\text { SDS } \\
\text { LDE }\end{array}$ & $\begin{array}{l}16.790 \\
116.7980 \\
10\end{array}$ & $\begin{array}{l}0.007 \\
0.009 \\
0.097\end{array}$ & 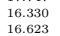 & .0.005 & $\begin{array}{l}16.344 \\
11.595\end{array}$ & 然.004 & $\begin{array}{l}16.401 \\
16.624 \\
16.62\end{array}$ & $\begin{array}{l}0.005 \\
0.005 \\
0\end{array}$ & 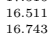 & $\begin{array}{l}0.008 \\
3 \\
0.009\end{array}$ & $\begin{array}{l}16.027 \\
16.149 \\
16.19\end{array}$ & $\left\{\begin{array}{l}0.007 \\
0.008\end{array}\right.$ & 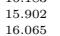 & $\begin{array}{l}0.013 \\
0.014\end{array}$ & $\begin{array}{l}15.886 \\
15.980\end{array}$ & $\begin{array}{l}0.018 \\
0.022\end{array}$ & $\begin{array}{l}\text { SDSS } \\
\text { CTIO }\end{array}$ \\
\hline 133 & 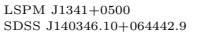 & $\begin{array}{l}1342421.80+050005458 \\
14034609+064434.0\end{array}$ & $\begin{array}{l}0.438 \\
0.131\end{array}$ & $\begin{array}{l}271.6 \\
155.2 \\
2\end{array}$ & $\begin{array}{l}\text { LEP } \\
\text { SDS }\end{array}$ & $\begin{array}{l}19.667 \\
18.028 \\
g\end{array}$ & $\begin{array}{l}0.035 \\
0.013\end{array}$ & $\begin{array}{l}17.413 \\
17.544\end{array}$ & 0.0 .005 & $\begin{array}{l}16.290 \\
17.274\end{array}$ & & $\begin{array}{l}15.854 \\
17.260\end{array}$ & $\begin{array}{l}0.004 \\
0.006 \\
0\end{array}$ & 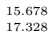 & 0006 & 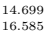 & & $\begin{array}{l}14.592 \\
16.625\end{array}$ & $\begin{array}{l}0.051 \\
0.276\end{array}$ & $\begin{array}{l}1.4 .78 \\
16.362\end{array}$ & 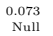 & $\begin{array}{l}\text { APO } \\
\text { DDSS }\end{array}$ \\
\hline & $\begin{array}{l}\text { SDSS } \\
\text { SDSS }\end{array}$ & 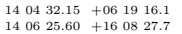 & $\begin{array}{l}0.105 \\
0.131\end{array}$ & $\begin{array}{l}117.8 \\
248.1\end{array}$ & $\begin{array}{l}\text { SDS } \\
\text { SDS }\end{array}$ & & & $\begin{array}{l}17.375 \\
17.610\end{array}$ & & & & & & & & & & & & $\begin{array}{l}16.445 \\
7.281\end{array}$ & 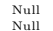 & $\begin{array}{l}\text { CTIO } \\
\text { SDSS }\end{array}$ \\
\hline & & $141039.98+024513.2$ & 0.237 & $\begin{array}{l}787.0 \\
187.0 \\
-1030\end{array}$ & SDS & $\begin{array}{l}18.087 \mathrm{O} \\
18.28\end{array}$ & .0.027 & $\begin{array}{l}17.034 \\
17.039 \\
1.509\end{array}$ & 0.019 & $\begin{array}{l}16.48 \\
16.48 \\
16.9\end{array}$ & & 6.328 & & & & & & .137 & & 15.015 & 0.158 & Is \\
\hline & & $\begin{array}{l}134.26+2 \\
218.79+0\end{array}$ & & & $\begin{array}{l}\text { SDS } \\
\text { sDs } \\
\text { sep }\end{array}$ & $\begin{array}{l}11.8948 \\
16.683 \\
\end{array}$ & & & & & & & & & & & & & & $\begin{array}{l}6.990 \\
5.871 \\
-10\end{array}$ & & \\
\hline & & & & & & & & & & & & & & & & & & & & & & $\begin{array}{l}\text { DSS } \\
\text { TIO }\end{array}$ \\
\hline & $77+244$ & & & & & & & & & & & & & & & & & & & & & \\
\hline
\end{tabular}


Table 1-Continued

\begin{tabular}{|c|c|c|c|c|c|c|c|c|c|c|c|c|c|c|c|c|c|c|}
\hline $\begin{array}{l}\text { WD } \\
\text { Name } \\
\end{array}$ & $\begin{array}{c}\text { Alternate } \\
\text { Name }\end{array}$ & R.A. (J2000.0) Decl. & 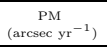 & $\begin{array}{l}\text { P.A. } \\
\text { (deg) }\end{array}$ & $\begin{array}{l}\text { PM } \\
\text { Ref. }\end{array}$ & $u$ & $\sigma_{u}$ & $\sigma_{g}$ & $\sigma_{r}$ & $\sigma_{i}$ & $\sigma_{z}$ & $J^{\prime}$ & $\sigma_{J}$ & $H$ & $\sigma_{H}$ & $K_{s}$ & $\sigma_{K_{s}}$ & Source \\
\hline 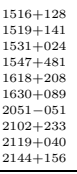 & 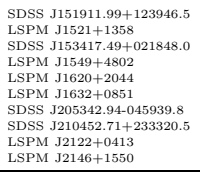 & 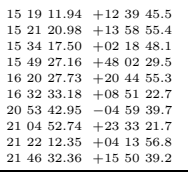 & $\begin{array}{l}0.113 \\
0.1168 \\
0.146 \\
0.176 \\
0.304 \\
0.376 \\
0.175 \\
0.139 \\
0.420 \\
0.330 \\
0\end{array}$ & 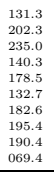 & $\begin{array}{l}\text { SDS } \\
\text { LEP } \\
\text { SDS } \\
\text { LEP } \\
\text { LEP } \\
\text { SDS } \\
\text { SDS } \\
\text { SDS } \\
\text { SDS } \\
\text { LEP } \\
\end{array}$ & 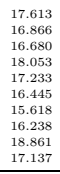 & $\begin{array}{l}0.012 \\
0.012 \\
0.000 \\
0.007 \\
0.015 \\
0.010 \\
0.007 \\
0.005 \\
0.006 \\
0.022 \\
0.010 \\
0.010 \\
\end{array}$ & 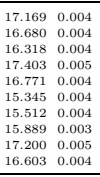 & 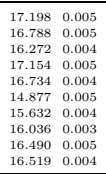 & 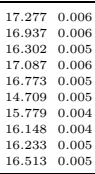 & 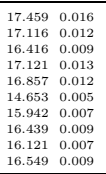 & 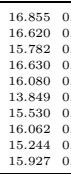 & 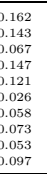 & $\begin{array}{l}16.472 \\
16.760 \\
15.792 \\
15.850 \\
15.980 \\
13.61 \\
15.652 \\
16.071 \\
15.009 \\
15.716 \\
\end{array}$ & $\begin{array}{l}0.270 \\
\text { Null } \\
0.126 \\
\text { Null } \\
0.230 \\
0.029 \\
0.145 \\
0.184 \\
0.070 \\
0.152 \\
0\end{array}$ & $\begin{array}{l}16.374 \\
16.190 \\
15.55 \\
16.750 \\
16.890 \\
13.48 \\
15.332 \\
16.200 \\
14.8276 \\
15.530\end{array}$ & $\begin{array}{ll}0.404 \\
\text { Null } \\
\text { Null } \\
\text { Null } \\
\text { Null } \\
\text { No.33 } \\
0.160 \\
\text { Null } \\
\text { Null } \\
\text { Null } \\
\text { Null }\end{array}$ & $\begin{array}{l}\text { SDSS } \\
\text { CDIO } \\
\text { SDSS } \\
\text { SDSS } \\
\text { SDSS } \\
\text { APO } \\
\text { APO } \\
\text { APO } \\
\text { APO } \\
\text { APO }\end{array}$ \\
\hline \multicolumn{19}{|c|}{ Spectroscopically Confirmed Subdwarfs } \\
\hline & 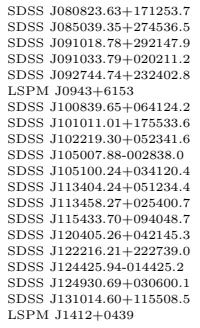 & 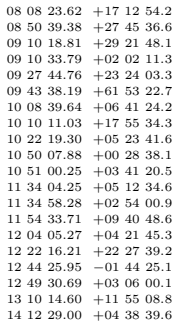 & 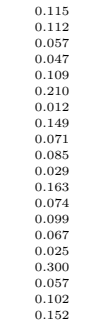 & 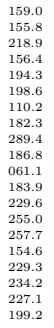 & $\begin{array}{l}\text { SSS } \\
\text { SSS } \\
\text { SSS } \\
\text { SSSS } \\
\text { LEP } \\
\text { SSS } \\
\text { SSS } \\
\text { SSS } \\
\text { SSS } \\
\text { SSS } \\
\text { SSS } \\
\text { SSS } \\
\text { SSS } \\
\text { SSS } \\
\text { SSS } \\
\text { SDS } \\
\text { LEP }\end{array}$ & 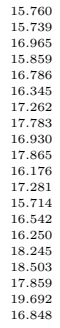 & 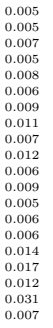 & 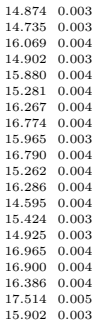 & 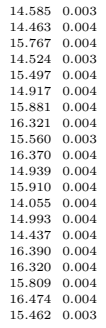 & 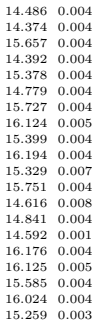 & 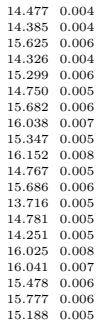 & 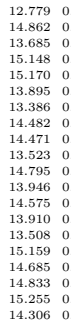 & 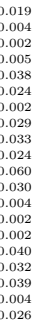 & 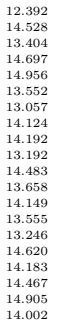 & 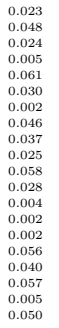 & 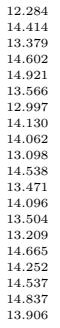 & 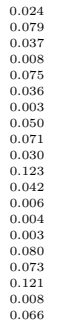 & 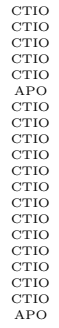 \\
\hline
\end{tabular}


TABLE 2

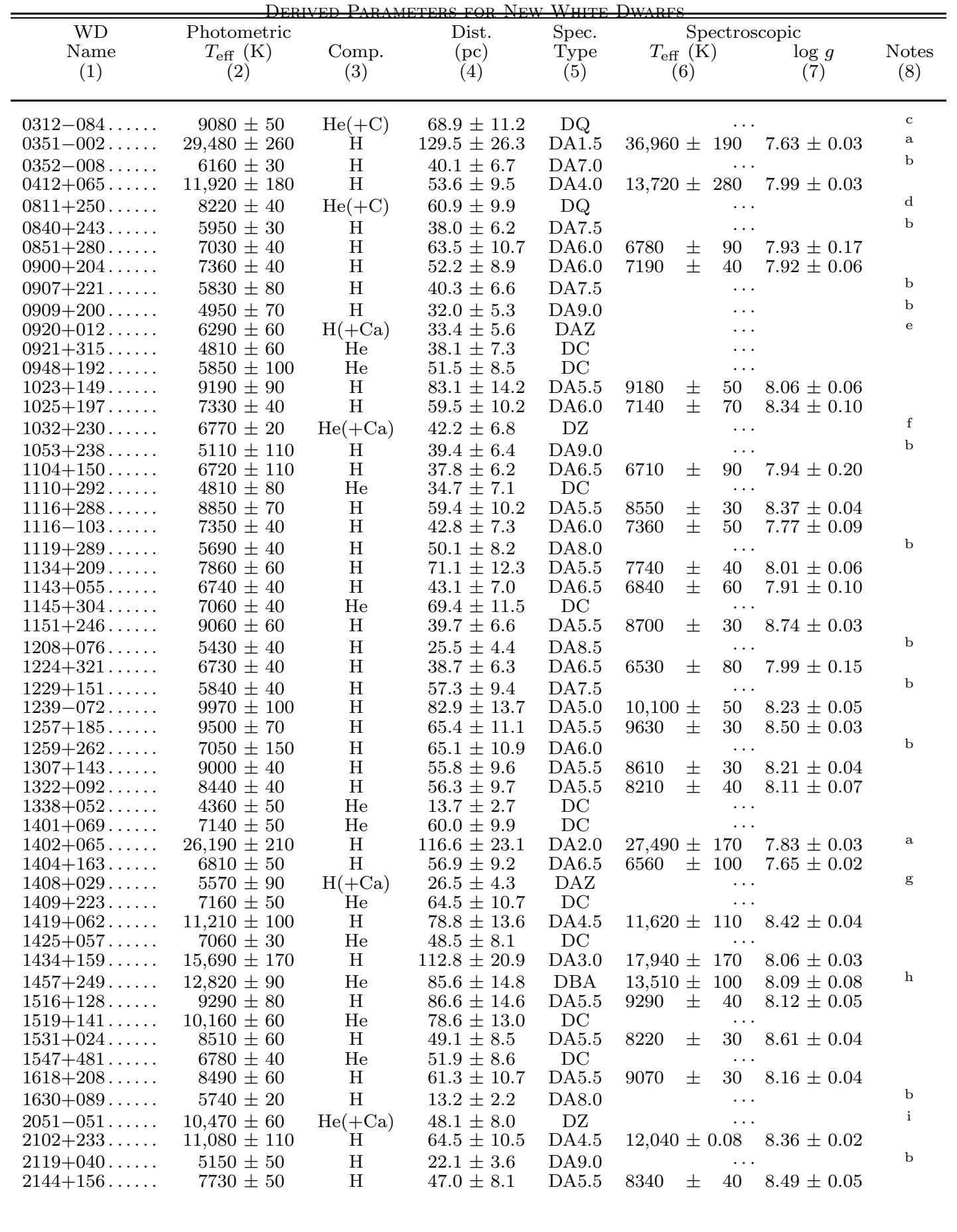

${ }^{2} T_{\text {eff }}$ from the SED fit is unreliable for the hottest WDs.

b A combination of weak Balmer lines and/or noisy spectra prohibited a reliable spectral fit.

c Best fit model includes $\log (\mathrm{C} / \mathrm{He})=-4.2 \pm 0.2$.

$\mathrm{d}$ Best fit model includes $\log (\mathrm{C} / \mathrm{He})=-5.1 \pm 0.2$

e Best fit model includes $\log (\mathrm{Ca} / \mathrm{H})=-9.0 \pm 0.2$.

$\mathrm{f}$ Best fit model includes $\log (\mathrm{Ca} / \mathrm{He})=-9.5 \pm 0.2$

$\mathrm{g}$ Best fit model includes $\log (\mathrm{Ca} / \mathrm{H})=-9.0 \pm 0.2$.

$\mathrm{h}$ Best fit model includes $\log (\mathrm{H} / \mathrm{He})=-5.9 \pm 0.1$

i Best fit model includes log $(\mathrm{Ca} / \mathrm{He})=-10.5 \pm 0.2$. 
TABLE 3

Survey Summary

\begin{tabular}{|c|c|c|c|c|c|c|c|c|c|c|}
\hline $\begin{array}{l}\text { Samp. } \\
\text { (1) }\end{array}$ & $\begin{array}{c}\text { PM } \\
\text { Source } \\
(2)\end{array}$ & $\begin{array}{c}\mathrm{Sky}^{\mathrm{c}} \\
\% \\
(3)\end{array}$ & $\begin{array}{c}\text { All Objs. } \\
\text { N } \\
(4)\end{array}$ & $\begin{array}{c}\text { Selected }^{\mathrm{d}} \\
\mathrm{N} \\
(5)\end{array}$ & $\begin{array}{c}\text { \# Obs. } \\
\text { WDs } \\
(6)\end{array}$ & $\begin{array}{l}\text { \# Obs. } \\
\text { contam. } \\
(7)\end{array}$ & $\begin{array}{l}\text { \# SDSS } \\
\text { Spect. } \\
(8)\end{array}$ & $\begin{array}{c}\text { \# Publ. } \\
\text { WDs } \\
(9)\end{array}$ & $\begin{array}{l}\text { \# Publ. } \\
\text { OTHER } \\
\quad(10)\end{array}$ & $\begin{array}{c}\text { Remaining } \\
\mathrm{N} \\
(11)\end{array}$ \\
\hline Pass $1^{\mathrm{a}}$ & USNO & 23 & 97137 & 1028 & 19 & 18 & 12 & 105 & 15 & 859 \\
\hline Pass $2^{\mathrm{b}}$ & LSPM & 29 & 19408 & 190 & 6 & 2 & 16 & 71 & 9 & 86 \\
\hline
\end{tabular}

a Initial Constraints: PM $>0.10$ arcsec $\mathrm{yr}^{-1}$, Decl. $<+30$ deg., $g<19.5,2$ MASS match, SDSS DR 8 footprint

b Initial Constraints: PM $>0.15$ arcsec $\mathrm{yr}^{-1}$, Decl. $>0$ deg., $g<19.5$, 2MASS match, SDSS DR8 footprint

C Estimated by applying initial declination constraints to SDSS DR8 footprint.

d Target selection criteria (see section [2]: RPM cut, $J-K_{s}$ cut, model-adherence cut. 

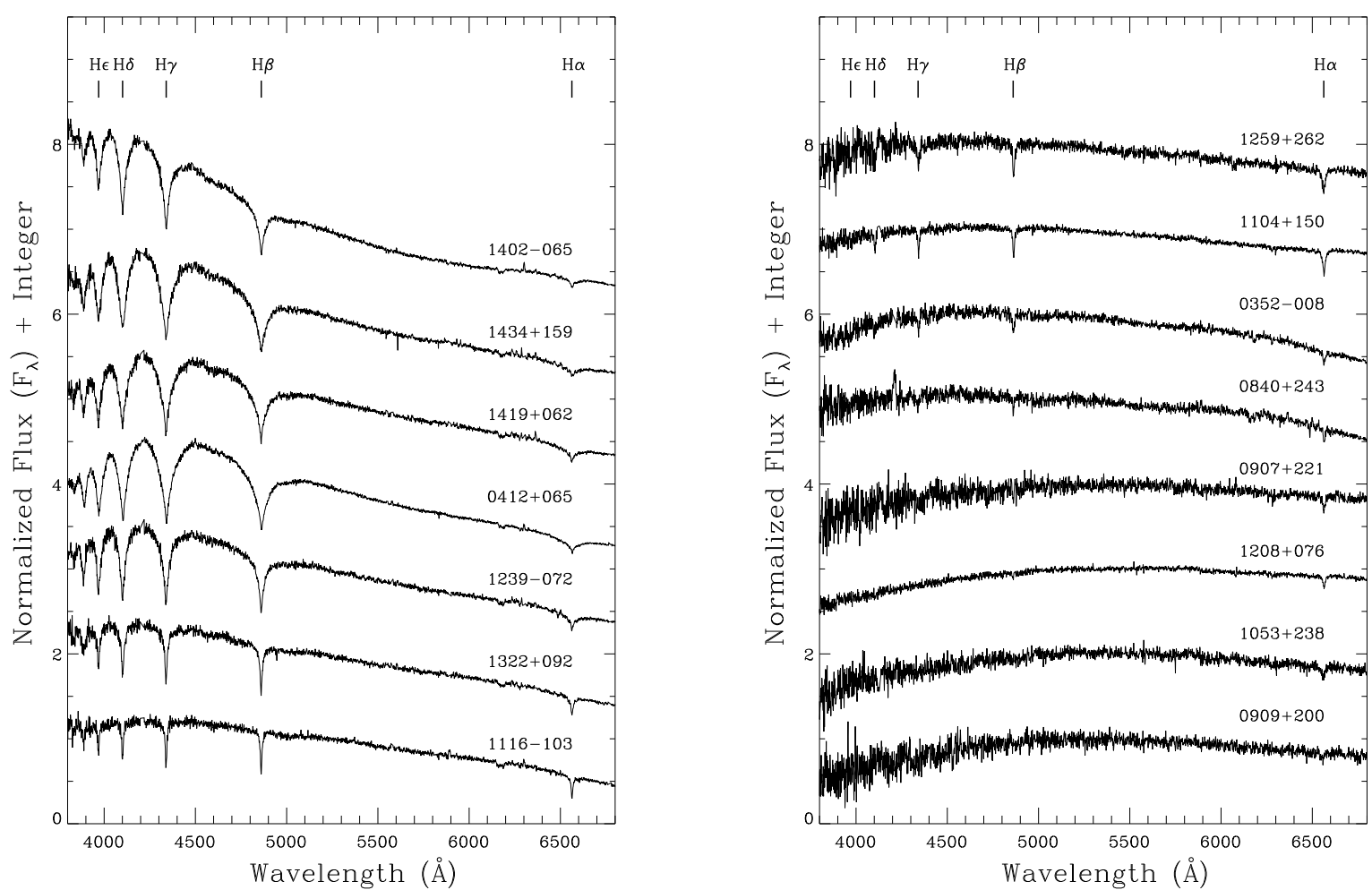

FIG. 1.- Hydrogen-line DA WDs, plotted in order of decreasing $T_{\text {eff }}$ from top to bottom. Balmer lines are indicated and WD designations are labeled above the spectra. WD $1419+062$ is included as discussed in Section 4.5 Spectra obtained at the CTIO $4 \mathrm{~m}$ Blanco Telescope. 
Sayres et al.

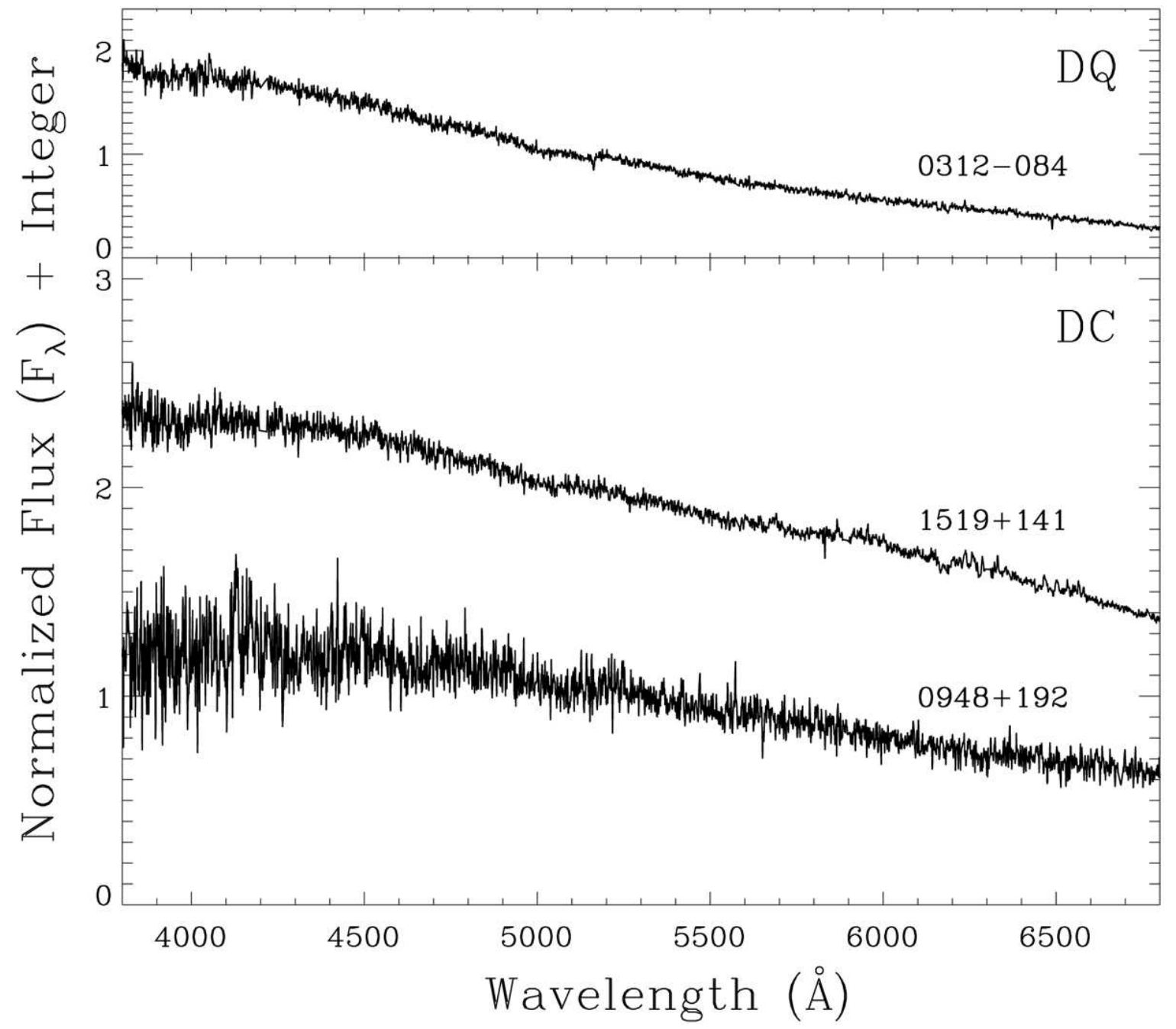

FIG. 2.- DQ (top panel) and DC (bottom panel) WDs, plotted in order of decreasing $T_{\text {eff }}$. Spectra obtained at the CTIO $4 \mathrm{~m}$ Blanco Telescope. 


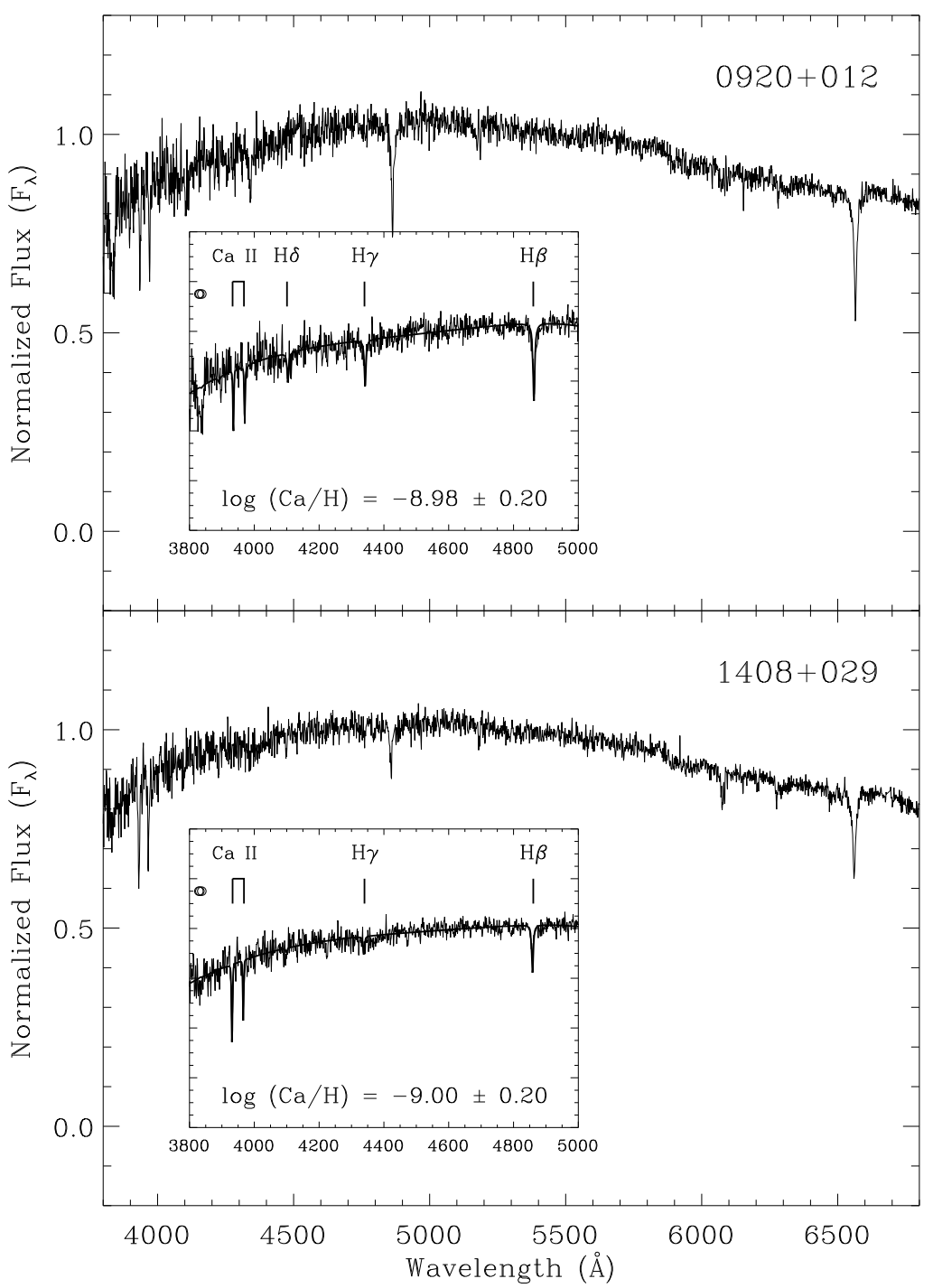

FIG. 3.- DAZ WDs with Ca II H \& K features. Model fits are displayed in the inset panels. Spectra obtained at the CTIO 4m Blanco Telescope. 

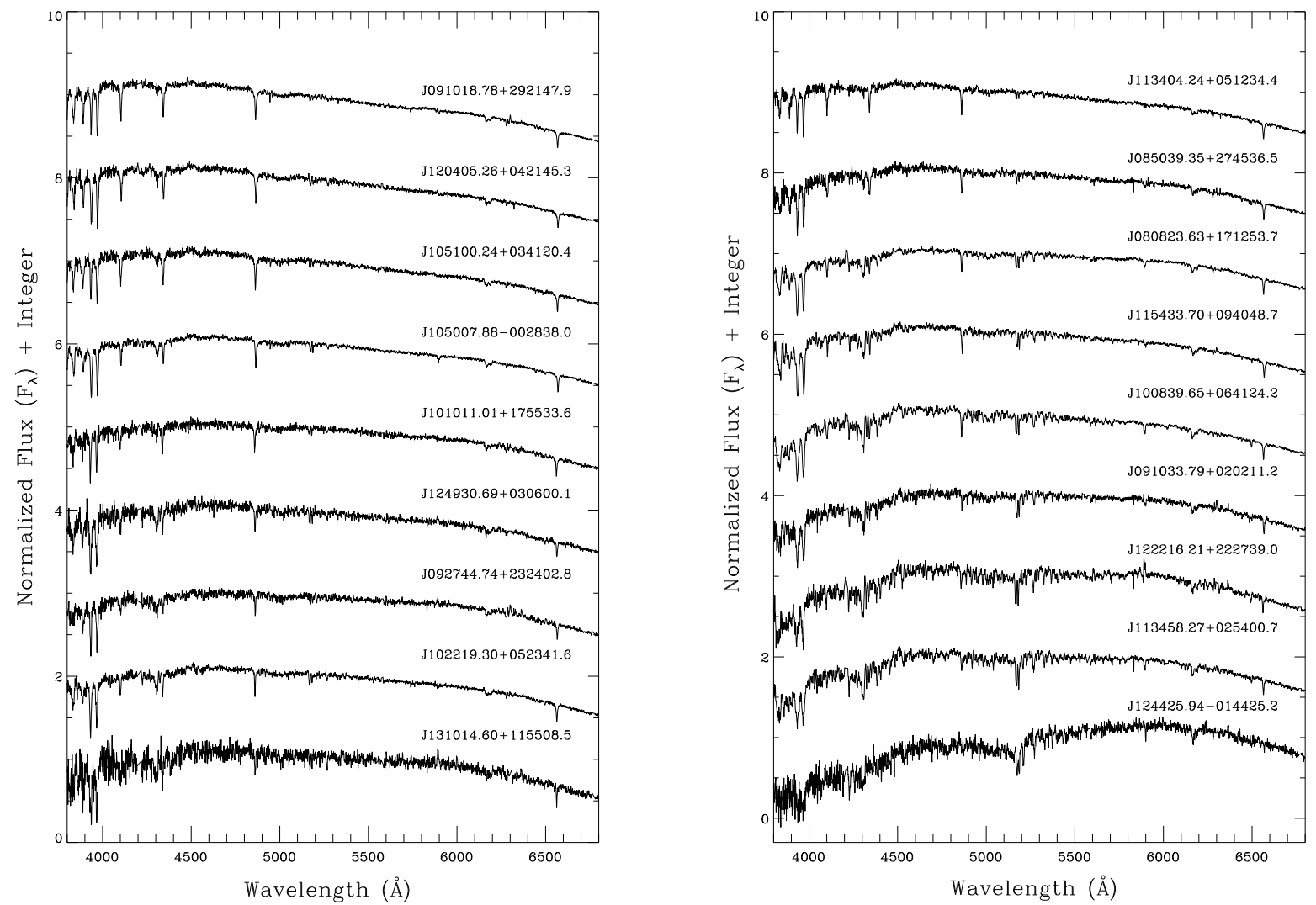

FIG. 4.- Contaminant spectra plotted in order of decreasing $T_{\text {eff }}$. Spectra obtained at the CTIO $4 \mathrm{~m}$ Blanco Telescope. 


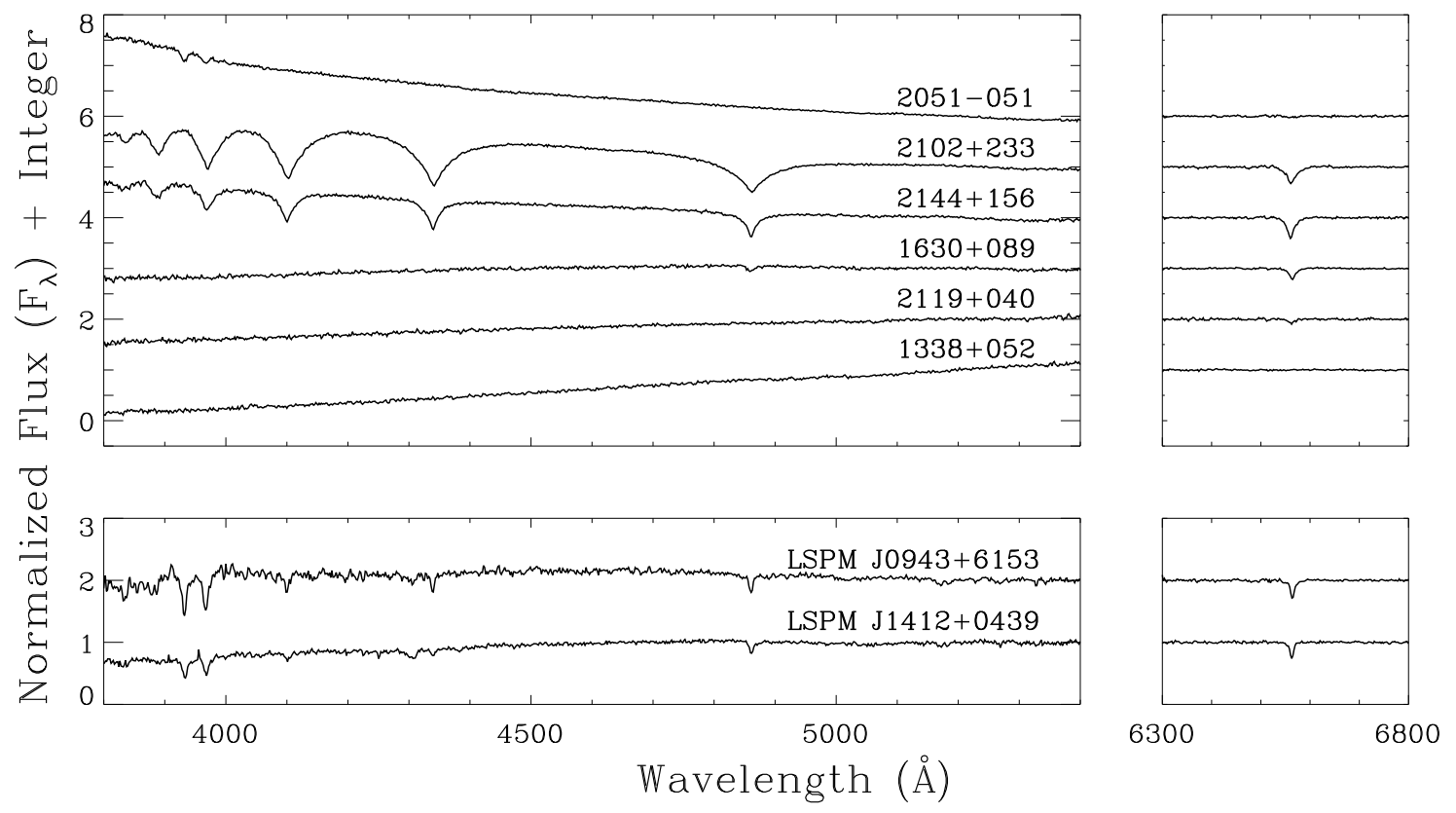

FIG. 5.- Spectra obtained from the ARC 3.5m Telescope at APO. Left panels represent the blue channel and right panels represent a subsection of the red channel centered on $\mathrm{H} \alpha$. Top panels are new WD discoveries while the bottom panels are two contaminants, likely SDs. All spectra are plotted in order of decreasing $T_{\text {eff }}$. 


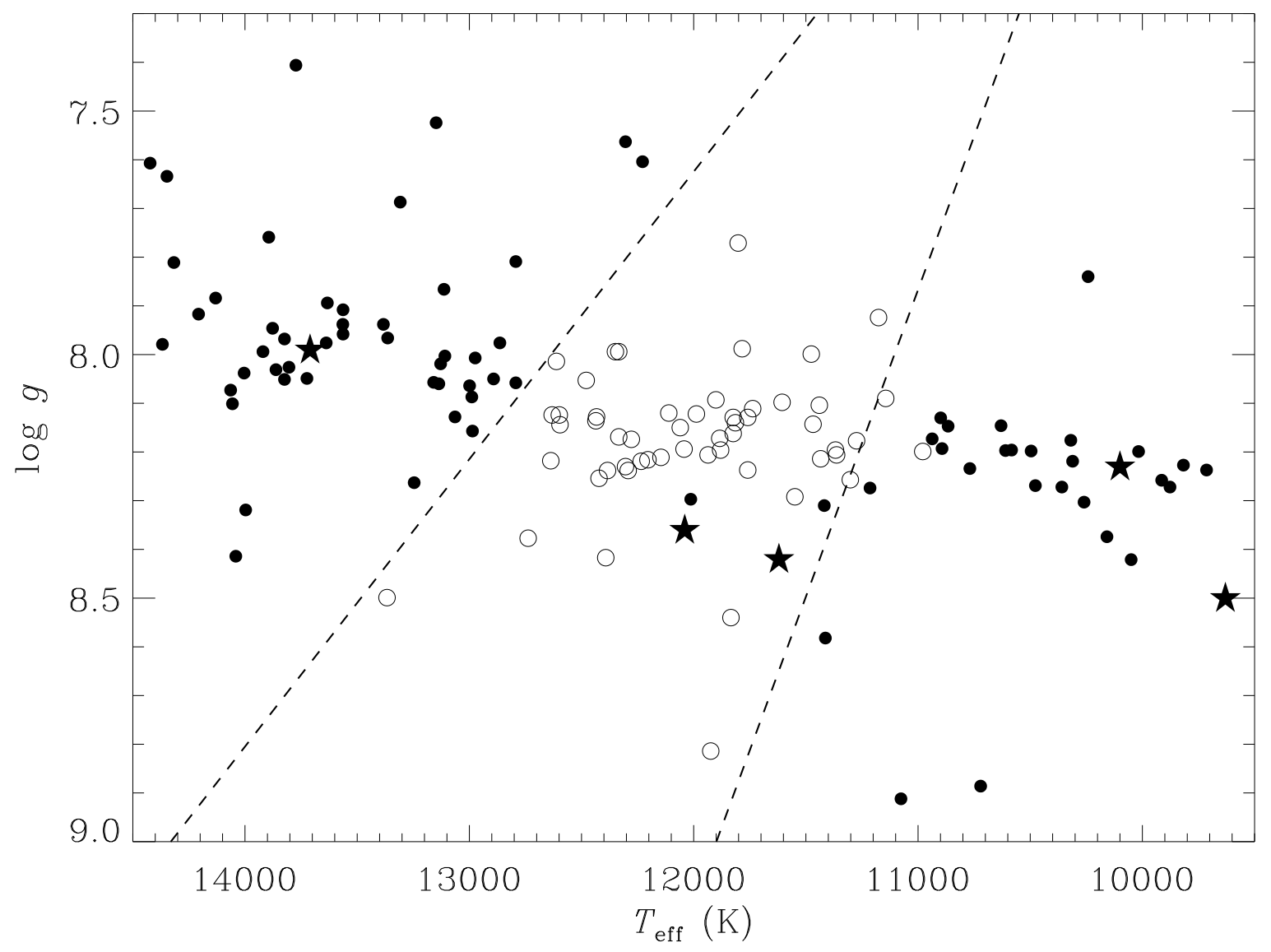

Fig. 6. - Plot of select new DA WDs with spectroscopic determinations of log $g$ and $T_{\text {eff }}$ (filled stars) - from left to right: 0412+065, $2102+233,1419+062,1239+072,1257+185$. Also plotted are WDs that are not observed to vary (filled circles) and ZZ Ceti confirmations (open circles). The dashed lines represent the instability strip boundaries as defined by Gianninas et al. (2011). 

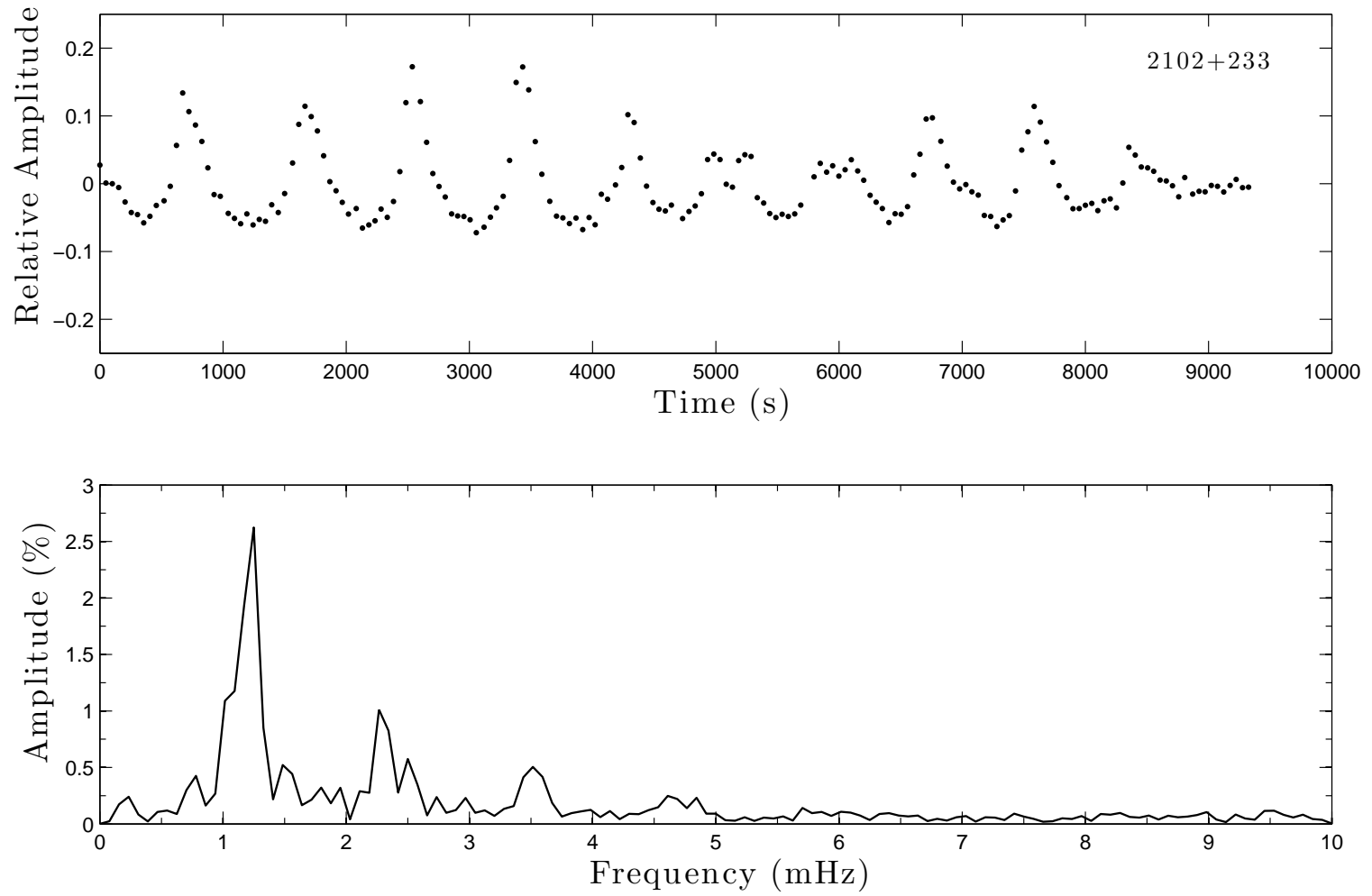

FIG. 7.- Light curve (top panel) and FT (bottom panel) for WD2102+233. Object was observed from the CTIO $0.9 \mathrm{~m}$ telescope at a cadence of $\sim 50$ seconds. From the FT, the dominant mode was found to be $\sim 800$ seconds 

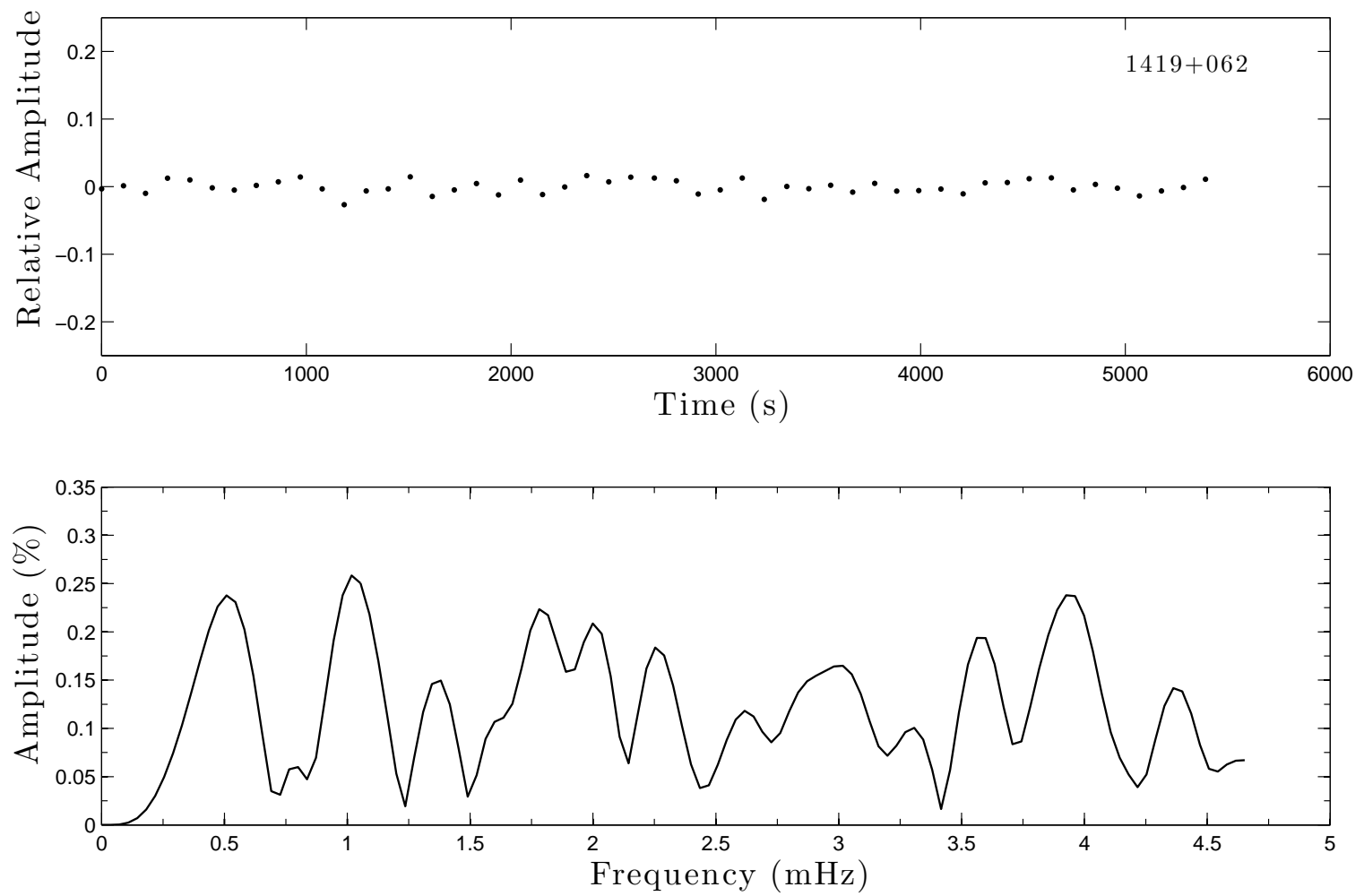

FIG. 8. - Light curve (top panel) and FT (bottom panel) for WD1419+062. Object was observed from the CTIO $1.0 \mathrm{~m}$ telescope at a cadence of $\sim 100$ seconds. From the FT, no dominant mode was observed above the noise level out to the Nyquist frequency. 


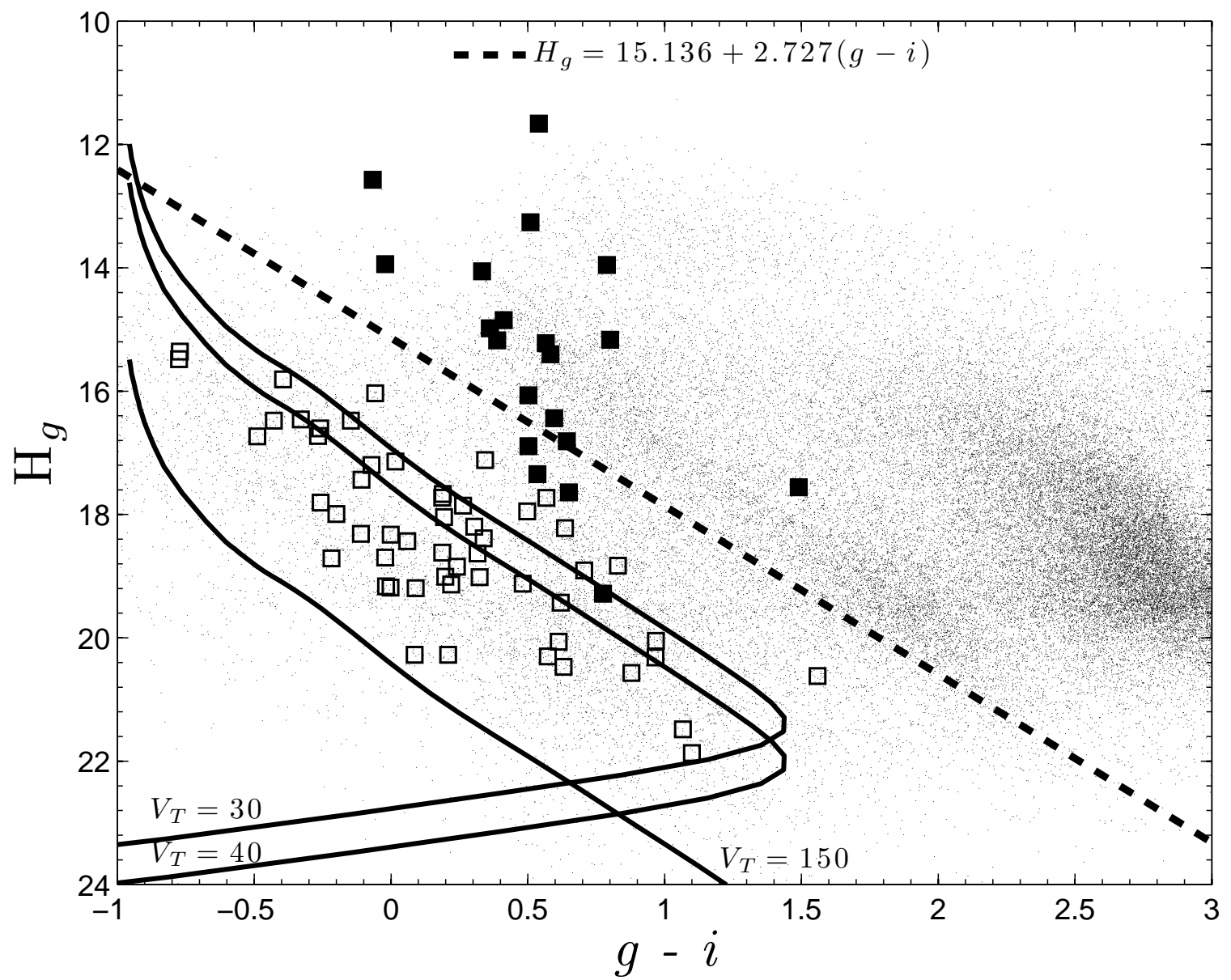

FIG. 9.- Reduced proper motion diagram showing WDs from this study (open squares) and contaminants (filled squares). The dashed line represents the cut adopted from Kilic et al. (2006) above which targets were discarded. Contaminants above the curve are discussed in Section 5 Modeled cooling curves for pure-hydrogen WDs with $\log g=8$ and $V_{\mathrm{tan}}=30,40$, and $150 \mathrm{~km} \mathrm{~s}^{-1}$ are plotted as solid curves. Background stars were taken from SDSS for reference. 